\title{
TIME REVERSAL OF WAVES IN A WEAKLY PERTURBED RANDOM MEDIUM*
}

\author{
DANIEL G. ALFARO VIGO ${ }^{\dagger}$ AND KNUT SØLNA
}

\begin{abstract}
In a time reversal experiment, a signal recorded by an array of transducers and sent back time-reversed into the same medium approximately refocuses on the original source center. The refocusing resolution is improved in an inhomogeneous medium. In this work we study the effect of changes in the medium, namely, the case when back propagation takes place in a perturbed medium.

Under the paraxial approximation assumption for a medium with weak inhomogeneities we consider a high frequency white noise regime. We show that relatively small perturbations do not affect the stable refocusing (self-averaging) for a localized source, but produce an interesting blurring of the refocused time signal. In some simple situations this effect can be explicitly quantified and related to the statistical model for the medium.
\end{abstract}

Key words. Random media, asymptotic theory, random Liouville equation, time reversal

AMS subject classifications. 35L05, 60H15, 35Q60

\section{Introduction}

In time reversal experiments a signal emitted by a localized source is recorded by an array of receiver-transducers [also known as a Time Reversal Mirror (TRM)]; then it is time-reversed and re-emitted into the medium; that is, the tail of the recorded signal is sent back first. In the absence of absorption the signal propagates back and focuses near the source. In Figure 1.1 a time reversal experiment is schematically illustrated. This phenomenon has numerous applications and has been thoroughly studied, experimentally and theoretically, see e.g., $[13,14,20,21]$. It has also been the subject of active mathematical research in the context of wave propagation in random media (some references relevant to this paper are mentioned below).

When time-reversal experiments are carried out in a (random) heterogeneous medium, under appropriate conditions, the size of the refocused spot appears to be smaller than in the case of a homogeneous medium. This surprising effect is a consequence of the multiple scattering in the inhomogeneous medium that creates multipathing and allows the transducer array to capture waves with a larger angular spread. The enhancement of the resolution of the refocused wave by the multipathing is called super-resolution [11]. Moreover, the time-reversed back-propagated pulse is also self-averaging so that the refocusing is statistically stable; that is, it does not depend on the particular realization of the random medium.

In the context of wave propagation in random media, these properties have been thoroughly studied under the paraxial approximation for several asymptotic regimes in $[4,11,17,18,19,30,31]$. The full wave equation was considered in $[5,6]$; see also the review [21]. The case with the full wave equation and layered random media is considered in $[22,23]$.

* Received: January 26, 2007; accepted (in revised version): February 23, 2008. Communicated by Lenya Ryzhik. This work was partially supported by DARPA grant N00014-02-1-0603, ONR grant N00014-02-1-0090, NSF grant 0307011 and the Sloan Foundation, DGAV was also supported by the National Council for Scientific and Technological Development (CNPq, Brazil) through the Instituto do Milênio.

†Instituto de Matemática Pura e Aplicada, Estrada D. Castorina 110, Rio de Janeiro 22460-320, BRAZIL (dgalfaro@impa.br).

${ }^{\ddagger}$ Department of Mathematics, University of California at Irvine, 103 MST Building, Irvine, CA 92697-3875, USA (ksolna@math.uci.edu). 


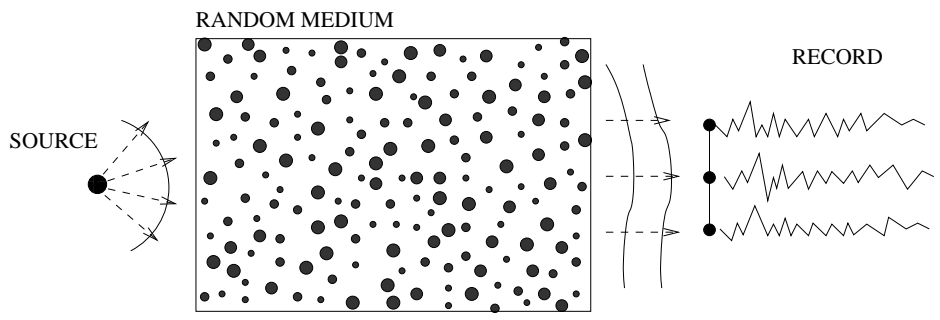

(a) PROPAGATION FORWARD

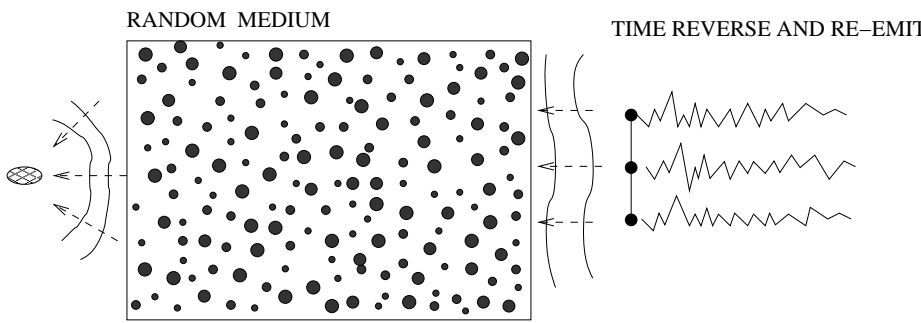

(b) BACK-PROPAGATION

FIG. 1.1. Schematic representation of a time reversal experiment in a random medium.

The aim of this work is to analyze how medium changes affect time reversal experiments. This is an important issue since in practical applications the medium properties may change during the experiment $[12,15]$. Some experimental studies have also shown that the refocused signal is modified as the underlying medium changes $[25,34]$. Therefore, we analyze time reversal experiments in a slightly different setting than the one described above. Namely, we allow the time-reversed wave to propagate back into a medium different from the one in which the forward propagation took place. [In the schematic representation in Figure 1.1, this means that the random medium in part (b) is different from the one in part (a).] This generalized time reversal procedure leads to more realistic models and presents a wider range of applications than the standard one [34].

Recently, time reversal with medium perturbations has been addressed theoretically and numerically. Bal \& Verástegui in [9] considered the transport and diffusive regimes for the full wave equation in two and three dimensions and in their theoretical study various asymptotics for the coherent time-reversed back-propagated wave were obtained. Moreover, their numerical experiments showed the statistical stabilization of the refocused wave.

The parabolic approximation has been used for the study of time reversal of waves since the pioneering paper of Blomgren, Papanicolaou \& Zhao (2002) [11]. For the parabolic wave approximation Bal \& Ryzhik presented in [7] a rigorous study of the effect of a changing medium. They considered the radiative transfer regime and the white noise limit, the Itô-Schrödinger regime, for the parabolic wave equation. In both cases, the effect of the changing medium is characterized as the combination of an effective absorption and phase modulation of the refocused signal at each frequency. They also established the statistical stabilization of the refocused wave.

Time reversal in the context of medium perturbations was studied in [1] for randomly layered media. The main result then states that the pulse shape is described by a random function, implying that the statistical stabilization property is lost, i.e., the 
refocused pulse is not statistically stable. In this paper we study the effect of medium perturbations on the quality of the refocused signal in the regime of the paraxial approximation in a scaling regime that is different from the one analyzed in [7]. We find again that the lateral medium variation, or diversity, then leads to a situation where the refocused signal is statistically stable also in the case with medium perturbations. We show that for a localized source relatively small perturbations in the medium do not affect the stable refocusing (self-averaging), but produce an interesting blurring of the refocused time signal. In some simple situations this effect can be explicitly quantified and related to the statistical model for the medium.

Some of the results obtained here are closely related to those of the recent paper [8]. There a diffusion limit for the expectation of the Wigner transform, corresponding to solutions of the Schrödinger equation, is analyzed. In the present work, besides the expectation we also study some second order statistics that allow us to establish the self-averaging of the back-propagated wave. Moreover, the Schrödinger equation treated here is non-autonomous, so we also analyze the effect of the lateral diversity that gives rise to a slightly different diffusion regime.

Our approach follows the same lines as in [30]; to obtain the self-averaging property we rely on the asymptotic analysis of characteristic ODEs corresponding to a random transport equation using the Kesten-Papanicolaou technique [27, 28] and some simple approximation arguments. We remark that there are several approaches to analyzing this problem and that another attractive technique involves directly establishing the asymptotic limit of the random PDE involved, as, for instance, in [16].

This paper is organized as follows: in Section 2 we describe the time reversal of waves in a perturbed medium, presenting the asymptotic regime we are interesting in and the mathematical description of the time-reversed and back-propagated wave. In Section 3, we consider the asymptotic behavior of the refocused wave. We describe the coherent time-reversed back-propagated limiting wave and establish its statistical stability. Finally, in Section 4 we interpret the results obtained and draw some concluding remarks. In Appendix A, some auxiliary results concerning the diffusion limit of random differential equations used in the main part of the paper are presented.

\section{Time reversal of waves in a perturbed medium}

In this section we generalize the analysis of time reversal of waves presented in [30] to the situation in which the medium has been subject to small perturbations.

2.1. Parabolic approximation and asymptotic regime. Recall that scalar wave propagation in a inhomogeneous medium is modeled by

$$
\frac{1}{c^{2}(\mathbf{x}, z)} \frac{\partial^{2} v}{\partial t^{2}}-\Delta_{(\mathbf{x}, z)} v=0
$$

where $c(\mathbf{x}, z)$ is the local wave speed, $\mathbf{x} \in \mathbb{R}^{d}$ with $d \geq 2$ is the transversal coordinate, $z$ represents the longitudinal coordinate, $t$ represents time, $\Delta_{(\mathbf{x}, z)}=\Delta+\frac{\partial^{2}}{\partial z^{2}}$ is the (full) Laplacian operator and $\Delta=\sum_{i=1}^{d} \frac{\partial^{2}}{\partial x_{i}^{2}}$ the transversal Laplacian.

Under the assumptions that the wave field has a 'beam-like' structure and that backscattering in the $z$ direction can be neglected, one can apply the parabolic or paraxial approximation [10] and write

$$
v(\mathbf{x}, z, t) \approx u(\mathbf{x}, z, t)=\int e^{i k\left(z-c_{0} t\right)} \psi(\mathbf{x}, z ; k) d k
$$


where the (complex) wave amplitude $\psi(\mathbf{x}, z ; k)$ is a solution of the Schrödinger equation

$$
2 i k \frac{\partial \psi}{\partial z}+\Delta \psi+k^{2}\left(n^{2}(\mathbf{x}, z)-1\right) \psi=0
$$

with suitable initial conditions and where $n(\mathbf{x}, z)=\frac{c_{0}}{c(\mathbf{x}, z)}$ represents the refraction index with respect to the reference speed $c_{0}$.

Next, we present a high-frequency regime for the parabolic approximation in which small scale separations take place. A closely related regime was introduced in [30] to analyze the statistical stabilization in time reversal of waves. Later on, we will analyze the asymptotic behavior of the wave field under medium perturbations in a successive limit.

Let $L_{x}, L_{z}$ be the characteristic propagation distance in the transversal and longitudinal directions, respectively, and $k_{0}$ the central carrier wave number of the incident wave. We introduce the scaling

$$
k=k_{0} k^{\prime}, \quad \mathbf{x}=L_{x} \mathbf{x}^{\prime}, \quad z=L_{z} z^{\prime} \quad \text { and } \quad n^{2}(\mathbf{x}, z)=1+2 \sigma \mu\left(\frac{\mathbf{x}}{l_{x}}, \frac{z}{l_{z}}\right)
$$

and the dimensionless parameters $\epsilon=\frac{l_{z}}{L_{z}}, \beta=\frac{1}{k_{0} l_{z}}$ and $\theta=\frac{L_{z}}{k_{0} L_{x}^{2}}$ (this is the inverse of the so-called Fresnel number). By letting $\frac{l_{x}}{L_{x}}=\epsilon^{a}$ and $\sigma=\frac{\beta \epsilon^{1 / 2+a}}{\theta}$, substitution in Equation (2.3) gives (after dropping primes) the scaled Schrödinger equation

$$
i k \theta \frac{\partial \psi}{\partial z}+\frac{1}{2} \theta^{2} \Delta \psi+k^{2} \mu^{\epsilon}(\mathbf{x}, z) \psi=0,
$$

where the fluctuations are given as

$$
\mu^{\epsilon}(\mathbf{x}, z)=\epsilon^{a-1 / 2} \mu\left(\frac{\mathbf{x}}{\epsilon^{a}}, \frac{z}{\epsilon}\right)
$$

with $\mu=O(1)$.

The regime that we are interested in appears when we assume the separation of scales

$$
\theta \ll \epsilon \ll 1
$$

and the conditions

$$
\beta \epsilon \ll \theta \text { and } \quad 0<a \leq 1,
$$

which ensures the validity of the parabolic approximation. The corresponding regime with $a=1$ was introduced in [30]. Concerning physical parameters these assumptions imply that

$$
\frac{1}{k_{0} l_{z}} \ll\left(\frac{L_{x}}{L_{z}}\right)^{2} \ll 1 \quad \text { and } \quad \frac{l_{x}}{L_{x}}=\left(\frac{l_{z}}{L_{z}}\right)^{a} \ll 1 .
$$

In the first condition, the first inequality corresponds to enforcing a high frequency regime, whereas the second inequality is the usual condition for validity of the paraxial approximation. The second condition states that the refractive index is anisotropic $(a<1)$ and fluctuates rapidly. Furthermore, the presence of a substantial lateral diversity is also enforced. 
The refractive index fluctuation $\sigma \mu(\cdot, \cdot)$ in Equation (2.4) will be modeled as a centered random field over $\mathbb{R}^{d}$ with $l_{x}, l_{z}$ corresponding to its transversal and longitudinal correlation lengths, respectively, and $\sigma$ to its mean square intensity.

Finally, we remark that in the dimensionless space variables introduced in (2.4) the approximated wave field $u^{\prime}\left(\mathbf{x}^{\prime}, z^{\prime}, t\right)=u\left(L_{x} \mathbf{x}^{\prime}, L_{z} z^{\prime}, t\right)$ reads (after dropping primes)

$$
u(\mathbf{x}, z, t)=k_{0} \int e^{i k k_{0}\left(L_{z} z-c_{0} t\right)} \psi(\mathbf{x}, z ; k) d k,
$$

where $\psi(\mathbf{x}, z ; k)$ is now the solution of Equation (2.5) with the corresponding initial conditions $\psi\left(\mathbf{x}, z=z_{0} ; k\right)=\psi_{I}(\mathbf{x} ; k)$. Furthermore, we have that

$$
\psi(\mathbf{x}, z ; k)=\int_{\mathbb{R}^{d}} G^{\theta, \epsilon}\left(\mathbf{x}, z ; \mathbf{y}, z_{0} ; k\right) \psi_{I}(\mathbf{y} ; k) d \mathbf{y}
$$

where $G^{\theta, \epsilon}$ is the Green's function corresponding to the Schrödinger Equation (2.5) which satisfies the initial condition $G^{\theta, \epsilon}\left(\mathbf{x}, z=z_{0} ; \mathbf{y}, z_{0} ; k\right)=\delta(\mathbf{x}-\mathbf{y})$.

2.2. Time-reversal of waves. In a standard time reversal experiment an emitted wave pulse is (partially) recorded by means of an array of receiver-transducers (Time-Reversal Mirror [TRM]); then it is time-reversed (corresponding to a phaseconjugation step) and re-emitted back into the medium. The main effect is the refocusing of this back-propagated wave near the location of the initial pulse. The refocusing is not exact because of the finite size of the TRM. It is well-known that the presence of inhomogeneities in the medium allows for a sharper focusing of the wave $[11,31]$; this property is called super-resolution. Furthermore, despite the fact that the refractive index is modeled as a random field, asymptotically the refocused wave does not depend on the random medium realization. This is the celebrated statistical stabilization (or self-averaging) property.

2.2.1. Medium perturbations. We further assume that the medium in which the wave propagates back after time reversal is different from the one of the forward propagation stage. More specifically, the refractive index fluctuations are given by the random fields $\mu_{f}^{\epsilon}(\mathbf{x}, z)$ and $\mu_{b}^{\epsilon}(\mathbf{x}, z)$, where the backward medium fluctuations are obtained by perturbations of the fluctuations in the forward medium, i.e., $\mu_{b}^{\epsilon}=\mu_{f}^{\epsilon}+\eta^{\epsilon}$. We shall analyze the case with perturbations such that $\eta^{\epsilon}=O\left(\frac{\theta}{\sqrt{\epsilon}}\right)$. Thus, we consider

$$
\begin{aligned}
& \mu_{f}^{\epsilon}(\mathbf{x}, z)=\epsilon^{a-1 / 2} \mu\left(\frac{\mathbf{x}}{\epsilon^{a}}, \frac{z}{\epsilon}\right), \\
& \eta^{\epsilon}(\mathbf{x}, z)=\frac{\theta}{\sqrt{\epsilon}} \eta\left(\frac{\mathbf{x}}{\epsilon^{a}}, \frac{z}{\epsilon}\right),
\end{aligned}
$$

where $\mu=O(1), \eta=O(1)$. Note that the perturbations are relatively small in the sense that $O\left(\eta^{\epsilon}(\cdot)\right) \ll O\left(\mu^{\epsilon}(\cdot)\right)$. We also assume that $\mu(\cdot, \cdot)$ and $\eta(\cdot, \cdot)$ are stationary, mean-zero, statistically independent, exponentially mixing and sufficiently smooth, isotropic random fields. More specifically, we assume that the isotropic random field $\left(\nabla_{\mathbf{x}} \mu(\cdot), \eta(\cdot)\right)^{t}$ satisfies conditions C.1-C.5 in Appendix A (on page 349).

We represent their covariances by

$$
\begin{aligned}
& R_{\mu}(\mathbf{x}, z)=\mathbb{E}\left\{\mu\left(\mathbf{x}+\mathbf{x}^{\prime}, z+z^{\prime}\right) \mu\left(\mathbf{x}^{\prime}, z^{\prime}\right)\right\}, \\
& R_{\eta}(\mathbf{x}, z)=\mathbb{E}\left\{\eta\left(\mathbf{x}+\mathbf{x}^{\prime}, z+z^{\prime}\right) \eta\left(\mathbf{x}^{\prime}, z^{\prime}\right)\right\}
\end{aligned}
$$


and their power spectra as

$$
\begin{aligned}
& \hat{R}_{\mu}(\mathbf{q}, r)=\int_{\mathbb{R}^{d+1}} e^{-i(\mathbf{x} \cdot \mathbf{q}+z \cdot r)} R_{\mu}(\mathbf{x}, z) d \mathbf{x} d z, \\
& \hat{R}_{\eta}(\mathbf{q}, r)=\int_{\mathbb{R}^{d+1}} e^{-i(\mathbf{x} \cdot \mathbf{q}+z \cdot r)} R_{\eta}(\mathbf{x}, z) d \mathbf{x} d z .
\end{aligned}
$$

2.2.2. Back-propagated wave. We consider a wave emitted from a localized source positioned at the point $\left(\mathbf{x}_{0}, 0\right)$; more specifically we assume initial conditions satisfying the scaling property

$$
\psi(\mathbf{x}, 0 ; k)=\psi_{I}(\mathbf{x} ; k)=\phi_{0}\left(\frac{\mathbf{x}-\mathbf{x}_{0}}{\theta} ; k\right) .
$$

The time-reversal mirror is located in the plane $z=L$. It is characterized by a mirror function $\chi(\mathbf{x})$. For instance, if the array of receiver-transducers is located in the domain $D \subset \mathbb{R}^{d}$ then the mirror function can be $\chi(\mathbf{x})=\mathbf{1}_{D}(\mathbf{x})$, the indicator function of the domain $D$, or a smooth function rapidly decaying outside $D$. We also model a blurring of the recorded signal by the mirror through a convolution kernel satisfying the scaling property $f_{\theta}(\mathbf{x})=\theta^{-d} f\left(\frac{\mathbf{x}}{\theta}\right)$, since the blurring should take place on the scale of the source $[4,5]$.

The back-propagated wave arriving at the plane $z=0$ and observed at the source location through a $\theta$-scaled window $u^{B}\left(\mathbf{x}_{0}+\theta \boldsymbol{\xi}, 0, t\right)$ will be expressed as

$$
u_{\theta}^{B}\left(\boldsymbol{\xi}, \mathbf{x}_{0}, t\right)=k_{0} \int e^{i k k_{0} c_{0} t} \phi_{\theta}^{B}\left(\boldsymbol{\xi}, \mathbf{x}_{0} ; k\right) d k
$$

where $\phi_{\theta}^{B}(\cdot)$ represents the corresponding (complex) wave amplitude. After several transformations we can rewrite, as in $[4,7]$ :

$$
\phi_{\theta}^{B}\left(\boldsymbol{\xi}, \mathbf{x}_{0}, k\right)=\int_{\mathbb{R}^{2 d}} e^{-i \mathbf{p} \cdot(\boldsymbol{\zeta}-\boldsymbol{\xi})} W^{\theta, \epsilon}\left(\mathbf{x}_{0}+\frac{\theta}{2}(\boldsymbol{\xi}+\boldsymbol{\zeta}), 0, \mathbf{p} ; k\right) \phi_{0}(\boldsymbol{\zeta} ; k) d \mathbf{p} d \boldsymbol{\zeta}
$$

and

$$
\begin{array}{r}
u_{\theta}^{B}\left(\boldsymbol{\xi}, \mathbf{x}_{0}, t\right)=k_{0} \iint_{\mathbb{R}^{2 d}} e^{i\left(k k_{0} c_{0} t-\mathbf{p} \cdot(\boldsymbol{\zeta}-\boldsymbol{\xi})\right)} W^{\theta, \epsilon}\left(\mathbf{x}_{0}+\frac{\theta}{2}(\boldsymbol{\xi}+\boldsymbol{\zeta}), 0, \mathbf{p} ; k\right) \\
\phi_{0}(\boldsymbol{\zeta} ; k) d \mathbf{p} d \boldsymbol{\zeta} d k,
\end{array}
$$

where we have introduced the 'mixed' Wigner transform

$$
W^{\theta, \epsilon}(\mathbf{x}, z, \mathbf{p} ; k)=\int_{\mathbb{R}^{d}} \hat{f}(\mathbf{q}) U^{\theta, \epsilon}(\mathbf{x}, z, \mathbf{p} ; \mathbf{q} ; k) d \mathbf{q}
$$

associated with the (pure state) Wigner transform on the scale $\theta$ (see details in [24, 29])

$$
U^{\theta, \epsilon}(\mathbf{x}, z, \mathbf{p} ; \mathbf{q} ; k)=\frac{1}{(2 \pi)^{d}} \int_{\mathbb{R}^{d}} e^{i \mathbf{p} \cdot \zeta} \overline{Q_{f}^{\theta, \epsilon}\left(\mathbf{x}+\frac{\theta}{2} \boldsymbol{\zeta}, z ; \mathbf{q} ; k\right)} Q_{b}^{\theta, \epsilon}\left(\mathbf{x}-\frac{\theta}{2} \boldsymbol{\zeta}, z ; \mathbf{q} ; k\right) d \boldsymbol{\zeta}
$$

corresponding to the family of functions $Q_{j}^{\theta, \epsilon}(\mathbf{x}, z ; \mathbf{q} ; k)$ representing the solutions of Equation (2.5) with final conditions $Q_{j}^{\theta, \epsilon}(\mathbf{x}, z=L ; \mathbf{q} ; k)=\chi(\mathbf{y}) e^{-i \mathbf{q} \frac{\mathbf{x}}{\theta}}$ when $\mu^{\epsilon}=\mu_{j}^{\epsilon}$, for $j=f, b$, respectively. 


\section{Asymptotics for the back-propagated wave}

We shall use asymptotic techniques $[24,30]$ to get information on the behavior of the wave field. We will perform two limiting processes: the first one, when $\theta$ goes to zero, corresponds to the geometrical optics limit (here randomness plays no role); In the second we let $\epsilon$ go to zero. This second step takes advantage of the random formulation, and we can readily identify the limiting averaged wave (coherent wave). Furthermore, we will establish that the limiting wave does not depend on the realizations of the refractive index fluctuations and coincides with the coherent backpropagated wave. This is the statistical stabilization or self-averaging property, and in our case it is a consequence of the decorrelation of the limiting Wigner transform at different wave vectors, as in the case of standard time reversal [11, 30].

3.1. Limiting back-propagated wave. Important statistical information on the back-propagated wave can be obtained by considering the asymptotics of its coherent component. In the regime we are analysing here, this reduces to the calculation of the limits

$$
\begin{aligned}
\bar{\phi}^{B}\left(\boldsymbol{\xi}, \mathbf{x}_{0} ; k\right) & =\lim _{(\theta \ll \epsilon) \rightarrow 0} \mathbb{E}\left\{\phi_{\theta}^{B}\left(\boldsymbol{\xi}, \mathbf{x}_{0} ; k\right)\right\}, \\
\bar{u}^{B}\left(\boldsymbol{\xi}, \mathbf{x}_{0}, t\right) & =\lim _{(\theta \ll \epsilon) \rightarrow 0} \mathbb{E}\left\{u_{\theta}^{B}\left(\boldsymbol{\xi}, \mathbf{x}_{0}, t\right)\right\},
\end{aligned}
$$

where by $\lim _{(\theta \ll \epsilon) \rightarrow 0}$ we mean the result of successively passing to the limit, as $\theta \rightarrow 0$ (with $\epsilon$ fixed) and after that as $\epsilon \rightarrow 0$.

According to the representations obtained in the previous section this leads us to the calculation of the limiting averaged Wigner transform

$$
W^{0}(\mathbf{x}, z=0, \mathbf{p} ; k)=\lim _{(\theta \ll \epsilon) \rightarrow 0} \mathbb{E}\left\{W^{\theta, \epsilon}(\mathbf{x}, 0, \mathbf{p} ; k)\right\} .
$$

These limiting quantities are related through the equations

$$
\begin{aligned}
\bar{u}^{B}\left(\boldsymbol{\xi}, \mathbf{x}_{0}, t\right) & =k_{0} \int e^{i k k_{0} c_{0} t} \bar{\phi}^{B}\left(\boldsymbol{\xi}, \mathbf{x}_{0} ; k\right) d k \\
\bar{\phi}^{B}\left(\boldsymbol{\xi}, \mathbf{x}_{0} ; k\right) & =\int_{\mathbb{R}^{d}} e^{-i \mathbf{p} \cdot \boldsymbol{\xi}} W^{0}\left(\mathbf{x}_{0}, 0, \mathbf{p} ; k\right) \tilde{\phi}_{0}(\mathbf{p} ; k) d \mathbf{p}
\end{aligned}
$$

where $\tilde{\phi}_{0}(\mathbf{p} ; k)$ represents the Fourier transform of $\phi_{0}(\mathbf{x} ; k)$ with respect to $\mathbf{x}$. Hence the computation of the coherent limiting wave is reduced to the calculation of the limiting averaged Wigner transform.

To obtain more detailed information one should proceed to the computation of higher order statistical moments of the back-propagated wave and its amplitude. However, in the present case, we have that the limiting wave amplitude and the back-propagated wave are statistically stable (or self-averaging), i.e., that their limits coincide with their averages.

In the next sections we carry out the corresponding asymptotic analysis in order to rigorously establish the mentioned properties and clearly state in which sense the convergence outlined above holds.

3.2. Wigner transform and the high frequency limit. Recall that the 'pure' Wigner transform $U^{\theta, \epsilon}$ associated to the functions $Q_{f}^{\theta, \epsilon}$ and $Q_{b}^{\theta, \epsilon}$ are bounded in a closed subspace of the (Schwartz) space of tempered distributions $\mathcal{S}^{\prime}\left(\mathbb{R}_{\mathbf{x}}^{d} \times \mathbb{R}_{\mathbf{p}}^{d}\right)$. Moreover, the corresponding 'mixed' Wigner transform $W^{\theta, \epsilon}$ appears as more regular; 
it is actually bounded in $L^{2}\left(\mathbb{R}_{\mathbf{x}}^{d} \times \mathbb{R}_{\mathbf{p}}^{d}\right)$ (see [29]). This extra regularity, which comes from the blurring effect of the mirror, has been used to establish some interesting results for Wigner transforms in random media (see for instance $[3,4]$ ). We will also take advantage of this property.

The Wigner transform $W^{\theta, \epsilon}(\mathbf{x}, z, \mathbf{p} ; k)$ satisfies, in the sense of distributions, the following Equation (see [24, 29]):

$$
\begin{aligned}
k \frac{\partial W^{\theta, \epsilon}}{\partial z}+\mathbf{p} \cdot \nabla_{\mathbf{x}} W^{\theta, \epsilon}= & \frac{i k^{2}}{(2 \pi)^{d} \theta} \int_{\mathbb{R}^{d}} e^{i \mathbf{q} \cdot \mathbf{x}}\left(\tilde{\mu}_{b}^{\epsilon}(\mathbf{q}, z) W^{\theta, \epsilon}\left(\mathbf{x}, z, \mathbf{p}-\frac{\theta}{2} \mathbf{q} ; k\right)\right. \\
& \left.-\tilde{\mu}_{f}^{\epsilon}(\mathbf{q}, z) W^{\theta, \epsilon}\left(\mathbf{x}, z, \mathbf{p}+\frac{\theta}{2} \mathbf{q} ; k\right)\right) d \mathbf{q}
\end{aligned}
$$

with the final condition

$$
W^{\theta, \epsilon}(\mathbf{x}, z=L, \mathbf{p} ; k)=W_{L}^{\theta}(\mathbf{x}, \mathbf{p})=\frac{1}{(2 \pi)^{d}} \int_{\mathbb{R}^{d}} e^{i \mathbf{p} \cdot \boldsymbol{\zeta}} f(\boldsymbol{\zeta}) \chi\left(\mathbf{x}+\frac{\theta}{2} \boldsymbol{\zeta}\right) \chi\left(\mathbf{x}-\frac{\theta}{2} \boldsymbol{\zeta}\right) d \boldsymbol{\zeta},
$$

where $\tilde{g}(\mathbf{q}, z)$ represents the (partial) Fourier transform of $g(\mathbf{x}, z)$ with respect to $\mathbf{x}$.

Equation (3.3) preserves the $L^{2}$-norm (this can be established as in [29]). Therefore, $W^{\theta, \epsilon}(\mathbf{x}, z, \mathbf{p} ; k)$ is uniformly bounded in $L^{2}\left(\mathbb{R}_{\mathbf{x}}^{d} \times \mathbb{R}_{\mathbf{p}}^{d}\right.$ ) (with respect to $\theta, \epsilon$ and the randomness).

It was proved in [4] that $W^{\theta, \epsilon}(\mathbf{x}, L, \mathbf{p} ; k) \rightarrow W_{L}(\mathbf{x}, \mathbf{p} ; k)$ (strongly) in $L^{2}\left(\mathbb{R}_{\mathbf{x}}^{d} \times \mathbb{R}_{\mathbf{p}}^{d}\right)$, where

$$
W_{L}(\mathbf{x}, \mathbf{p} ; k)=\hat{f}(\mathbf{p}) \chi^{2}(\mathbf{x}) .
$$

As a consequence, in the (random) geometrical optics limit (i.e., when $\theta \rightarrow 0$ for each fixed realization of the random field), for any sequence $\theta_{n}$ (after possibly extracting a subsequence) $W^{\theta_{n}, \epsilon}$ converges (strongly) in $L^{2}\left(\mathbb{R}_{\mathbf{x}}^{d} \times \mathbb{R}_{\mathbf{p}}^{d}\right)$ as $\theta_{n} \rightarrow 0$. Furthermore, a straightforward calculation shows that its limit $W^{\epsilon}$ satisfies the following (random) transport equation [24, 29]:

$$
k \frac{\partial W^{\epsilon}}{\partial z}+\mathbf{p} \cdot \nabla_{\mathbf{x}} W^{\epsilon}+\frac{k^{2}}{\sqrt{\epsilon}} \nabla_{\perp} \mu\left(\frac{\mathbf{x}}{\epsilon^{a}}, \frac{z}{\epsilon}\right) \cdot \nabla_{\mathbf{p}} W^{\epsilon}-\frac{i k^{2}}{\sqrt{\epsilon}} \eta\left(\frac{\mathbf{x}}{\epsilon^{a}}, \frac{z}{\epsilon}\right) W^{\epsilon}=0
$$

with final condition

$$
W^{\epsilon}(\mathbf{x}, z=L, \mathbf{p} ; k)=W_{L}(\mathbf{x}, \mathbf{p} ; k)=\hat{f}(\mathbf{p}) \chi^{2}(\mathbf{x}),
$$

where $\nabla_{\perp} \mu(\mathbf{x}, \cdot)=\nabla_{\mathbf{x}} \mu(\mathbf{x}, \cdot)$. Notice that Equation (3.5) preserves the $L^{2}$-norm; this implies uniqueness in $L^{2}$, and as a consequence the convergence as $\theta \rightarrow 0$ is guaranteed without choosing any subsequence. Additionally, the functions $W^{\epsilon}$ are uniformly bounded in $L^{2}\left(\mathbb{R}_{\mathbf{x}}^{d} \times \mathbb{R}_{\mathbf{p}}^{d}\right)$ (with respect to $\epsilon$ and the randomness).

The following theorem presents the high-frequency asymptotics of the backpropagated wave. In particular, it states that one can drop the term $\frac{\theta}{2}(\boldsymbol{\xi}+\boldsymbol{\zeta})$ in the argument of $W^{\theta, \epsilon}$ in expressions (2.17) and (2.18), and use the corresponding asymptotics of the Wigner transform.

THEOREM 3.1. (random geometrical optics asymptotics.) Let $\epsilon$ and the medium realization be fixed. Assume that the source function $\phi_{0} \in L^{2}\left(\mathbb{R}_{\zeta}^{d} \times \mathbb{R}_{k}\right)$. Then for every $\boldsymbol{\xi} \in \mathbb{R}^{d}$ and almost every $k$, the complex amplitude $\phi_{\theta}^{B}\left(\boldsymbol{\xi}, \mathbf{x}_{0} ; k\right)$ converges (strongly) in $L^{2}\left(\mathbb{R}_{\mathbf{x}_{0}}^{d}\right)$, as $\theta \rightarrow 0$, to the function

$$
\phi_{\epsilon}^{B}\left(\boldsymbol{\xi}, \mathbf{x}_{0} ; k\right)=\int_{\mathbb{R}^{d}} e^{-i \mathbf{p} \cdot \boldsymbol{\xi}} W^{\epsilon}\left(\mathbf{x}_{0}, 0, \mathbf{p} ; k\right) \tilde{\phi}_{0}(\mathbf{p} ; k) d \mathbf{p},
$$


where $W^{\epsilon}(\mathbf{x}, z, \mathbf{p} ; k)$ is the solution of Equation (3.5)-(3.6).

Furthermore, the back-propagated wave $u_{\theta}^{B}\left(\boldsymbol{\xi}, \mathbf{x}_{0}, t\right)$ converges (strongly) in $L^{2}\left(\mathbb{R}_{\mathbf{x}_{0}}^{d} \times \mathbb{R}_{t}\right)$ to

$$
u_{\epsilon}^{B}\left(\boldsymbol{\xi}, \mathbf{x}_{0}, t\right)=k_{0} \int e^{i k k_{0} c_{0} t} \phi_{\epsilon}^{B}\left(\boldsymbol{\xi}, \mathbf{x}_{0} ; k\right) d k
$$

Proof. In the first part of the proof, in order to simplify notation, we suppress the dependence on $k$. We have to prove that

$$
\int d \mathbf{x}\left|\int e^{-i \mathbf{p}(\boldsymbol{\xi}-\boldsymbol{\zeta})}\left(W^{\theta, \epsilon}\left(\mathbf{x}+\frac{\theta}{2}(\boldsymbol{\xi}+\boldsymbol{\zeta}), 0, \mathbf{p}\right)-W^{\epsilon}(\mathbf{x}, 0, \mathbf{p})\right) \phi_{0}(\boldsymbol{\zeta}) d \boldsymbol{\zeta} d \mathbf{p}\right|^{2} \rightarrow 0 .
$$

From the decomposition

$$
\begin{array}{r}
W^{\theta, \epsilon}\left(\mathbf{x}_{0}+\frac{\theta}{2}(\boldsymbol{\xi}+\boldsymbol{\zeta}), \cdot\right)-W^{\epsilon}\left(\mathbf{x}_{0}, \cdot\right)=W^{\theta, \epsilon}\left(\mathbf{x}_{0}+\frac{\theta}{2}(\boldsymbol{\xi}+\boldsymbol{\zeta}), \cdot\right)-W^{\epsilon}\left(\mathbf{x}_{0}+\frac{\theta}{2}(\boldsymbol{\xi}+\boldsymbol{\zeta}), \cdot\right) \\
+W^{\epsilon}\left(\mathbf{x}_{0}+\frac{\theta}{2}(\boldsymbol{\xi}+\boldsymbol{\zeta}), \cdot\right)-W^{\epsilon}\left(\mathbf{x}_{0}, \cdot\right),
\end{array}
$$

it follows that it is enough to prove the corresponding convergence result for each term of this decomposition.

Using the Fourier transform, we can write the integral corresponding to the first term as

$$
\begin{aligned}
I_{1}=\int d \mathbf{x} \mid \int e^{-i \mathbf{p}(\boldsymbol{\xi}-\boldsymbol{\zeta})}\left(W^{\theta, \epsilon}\left(\mathbf{x}+\frac{\theta}{2}(\boldsymbol{\xi}+\boldsymbol{\zeta}), 0, \mathbf{p}\right)\right. \\
\left.\quad-W^{\epsilon}\left(\mathbf{x}+\frac{\theta}{2}(\boldsymbol{\xi}+\boldsymbol{\zeta}), 0, \mathbf{p}\right)\right)\left.\phi_{0}(\boldsymbol{\zeta}) d \boldsymbol{\zeta} d \mathbf{p}\right|^{2} \\
=(2 \pi)^{d} \int d \mathbf{q}\left|\int e^{-i\left(\mathbf{p}-\frac{\theta}{2} \mathbf{q}\right) \boldsymbol{\xi}}\left(\tilde{W}^{\theta, \epsilon}(\mathbf{q}, 0, \mathbf{p})-\tilde{W}^{\epsilon}(\mathbf{q}, 0, \mathbf{p})\right) \hat{\hat{\phi}_{0}\left(\mathbf{p}+\frac{\theta}{2} \mathbf{q}\right)} d \mathbf{p}\right|^{2},
\end{aligned}
$$

and using the Cauchy-Schwartz inequality we get the estimate

$$
\begin{aligned}
\left|I_{1}\right| & \leq(2 \pi)^{d}\left\|\hat{\phi}_{0}\right\|_{L^{2}}^{2} \int\left|\tilde{W}^{\theta, \epsilon}(\mathbf{q}, 0, \mathbf{p})-\tilde{W}^{\epsilon}(\mathbf{q}, 0, \mathbf{p})\right|^{2} d \mathbf{q} d \mathbf{p} \\
& \leq\left\|\hat{\phi}_{0}\right\|_{L^{2}\left(\mathbb{R}_{\mathbf{p}}^{d}\right)}^{2}\left\|W^{\theta, \epsilon}(0, \cdot)-W^{\epsilon}(0, \cdot)\right\|_{L^{2}\left(\mathbb{R}_{\mathbf{x}}^{d} \times \mathbb{R}_{\mathbf{p}}^{d}\right)}^{2} \rightarrow 0 .
\end{aligned}
$$

For the integral corresponding to the second term, after using the Fourier transform, we have

$$
\begin{aligned}
I_{2} & =\int d \mathbf{x}\left|\int e^{-i \mathbf{p}(\boldsymbol{\xi}-\boldsymbol{\zeta})}\left(W^{\epsilon}\left(\mathbf{x}+\frac{\theta}{2}(\boldsymbol{\xi}+\boldsymbol{\zeta}), 0, \mathbf{p}\right)-W^{\epsilon}(\mathbf{x}, 0, \mathbf{p})\right) \phi_{0}(\boldsymbol{\zeta}) d \boldsymbol{\zeta} d \mathbf{p}\right|^{2} \\
& =(2 \pi)^{d} \int d \mathbf{q}\left|\int\left(e^{i \frac{\theta}{2}(\boldsymbol{\xi}+\boldsymbol{\zeta})}-1\right) \overline{\hat{W}^{\epsilon}(\mathbf{q}, 0, \boldsymbol{\zeta}-\boldsymbol{\xi})} \phi_{0}(\boldsymbol{\zeta}) d \boldsymbol{\zeta}\right|^{2} \\
& \leq(2 \pi)^{d} \int I_{\theta}^{2}(\mathbf{q}) d \mathbf{q}
\end{aligned}
$$

where

$$
I_{\theta}(\mathbf{q})=\int\left|\left(e^{i \frac{\theta}{2}(\boldsymbol{\xi}+\boldsymbol{\zeta})}-1\right)\right|\left|\overline{\hat{W}^{\epsilon}(\mathbf{q}, 0, \boldsymbol{\zeta}-\boldsymbol{\xi})} \phi_{0}(\boldsymbol{\zeta})\right| d \boldsymbol{\zeta} .
$$

Note that $I_{\theta}(\mathbf{q}) \rightarrow 0$ for almost every $\mathbf{q}$ as $\theta \rightarrow 0$. Indeed, its integrand converges to zero pointwise. Furthermore, it is bounded by $2\left|\overline{\hat{W}^{\epsilon}(\mathbf{q}, 0, \boldsymbol{\zeta}-\boldsymbol{\xi})} \phi_{0}(\boldsymbol{\zeta})\right|$, which is integrable 
with respect to $\zeta$ for almost every $\mathbf{q}$, as a direct consequence of the Cauchy-Schwartz inequality and the fact that $\hat{W}^{\epsilon}(0, \cdot) \in L^{2}\left(\mathbb{R}_{\mathbf{q}}^{d} \times \mathbb{R}_{\zeta}^{d}\right)$. By applying the dominated convergence theorem, the result follows.

On the other hand, using the Cauchy-Schwartz inequality, we have the estimate

$$
I_{\theta}^{2}(\mathbf{q}) \leq 4\left\|\phi_{0}\right\|_{L^{2}\left(\mathbb{R}_{\zeta}^{d}\right)}^{2}\left\|\hat{W}^{\epsilon}(\mathbf{q}, 0, \cdot)\right\|_{L^{2}\left(\mathbb{R}_{\zeta}^{d}\right)}^{2},
$$

and the function in the right hand side is integrable. Consequently, from the dominated convergence theorem we conclude that $I_{2} \rightarrow 0$ as $\theta \rightarrow 0$.

For the proof of the second part of the theorem we explicitly write the dependence on $k$.

We prove the equivalent statement that, as $\theta \rightarrow 0$,

$$
\int d k \int_{\mathbb{R}^{d}}\left|\phi_{\theta}^{B}(\boldsymbol{\xi}, \mathbf{x} ; k)-\phi_{\epsilon}^{B}(\boldsymbol{\xi}, \mathbf{x} ; k)\right|^{2} d \mathbf{x}=\int I(k) d k \rightarrow 0 .
$$

Note that the first part of the theorem states that $I(k) \rightarrow 0$, for $k \neq 0$. Furthermore, similarly as in that part of the proof, one can get the estimates

$$
|I(k)| \leq\left\|\phi_{0}(\cdot ; k)\right\|_{L^{2}\left(\mathbb{R}_{\zeta}^{d}\right)}^{2}\left(C_{1}\left\|W^{\theta, \epsilon}(0, \cdot ; k)\right\|_{L^{2}\left(\mathbb{R}_{\mathbf{x}}^{d} \times \mathbb{R}_{\mathbf{p}}^{d}\right)}^{2}+C_{2}\left\|W^{\epsilon}(0, \cdot ; k)\right\|_{L^{2}\left(\mathbb{R}_{\mathbf{x}}^{d} \times \mathbb{R}_{\mathbf{p}}^{d}\right)}^{2}\right),
$$

where $C_{1}, C_{2}$ are real constants. Additionally, since Equations (3.3) and (3.5) conserve the $L^{2}$-norm and $W_{L}^{\theta}$ does not depend on $k$, we get that there is a real constant $C_{3}$ such that $|I(k)| \leq C_{3}\left\|\phi_{0}(\cdot ; k)\right\|_{L^{2}\left(\mathbb{R}_{\zeta}^{d}\right)}^{2}$. Finally, to conclude the proof we use the dominated convergence theorem and the fact that $\phi_{0} \in L^{2}\left(\mathbb{R}_{\zeta}^{d} \times \mathbb{R}_{k}\right)$.

3.3. Diffusion limit for the Wigner transform. In this section, we characterize the limit as $\epsilon \rightarrow 0$ of the (random) geometrical optics Wigner transform $W^{\epsilon}$. More specifically, we prove that $\lim _{\epsilon \rightarrow 0} W^{\epsilon}(\mathbf{x}, z=0, \mathbf{p} ; k)=W^{0}(\mathbf{x}, z=0, \mathbf{p} ; k)$, where convergence is understood in the weak sense and the function $W^{0}$ solves a deterministic transport-diffusion equation. This highlights the self-averaging property of the Wigner transform.

3.3.1. Convergence of the expectation. We start by characterizing the asymptotics as $\epsilon \rightarrow 0$ of the averaged geometrical optics Wigner transform $E\left\{W^{\epsilon}(\mathbf{x}, z=0, \mathbf{p} ; k)\right\}$. This goal is achieved in part because of the 'white noise' scaling in the coefficients of Equation (3.5), their regularity and mixing properties.

Observe that the solution of Equation (3.5)-(3.6) has the representation

$$
W^{\epsilon}(\mathbf{x}, 0, \mathbf{p} ; k)=W_{L}\left(\mathbf{X}^{\epsilon}(L ; \mathbf{x}, \mathbf{p}), \mathbf{P}^{\epsilon}(L ; \mathbf{x}, \mathbf{p}) ; k\right) e^{-i Q^{\epsilon}(L ; \mathbf{x}, \mathbf{p})},
$$

where $\mathbf{X}^{\epsilon}(s)=\mathbf{X}^{\epsilon}(s ; \mathbf{x}, \mathbf{p}), \mathbf{P}^{\epsilon}(s)=\mathbf{P}^{\epsilon}(s ; \mathbf{x}, \mathbf{p}), Q^{\epsilon}(s)=Q^{\epsilon}(L ; \mathbf{X}, \mathbf{p})$ solves the characteristics ODEs corresponding to Equation (3.5),

$$
\left\{\begin{aligned}
\frac{d}{d s} \mathbf{X}^{\epsilon} & =\frac{\mathbf{P}^{\epsilon}}{k} \\
\frac{d}{d s}\left(\begin{array}{c}
\mathbf{P}^{\epsilon} \\
Q^{\epsilon}
\end{array}\right) & =\frac{k}{\sqrt{\epsilon}}\left(\begin{array}{c}
\nabla \perp \mu\left(\frac{\mathbf{X}^{\epsilon}}{\epsilon^{\epsilon}}, \frac{s}{\epsilon}\right) \\
\eta\left(\frac{\mathbf{X}^{\epsilon}}{\epsilon^{a}}, \frac{s}{\epsilon}\right)
\end{array}\right),
\end{aligned}\right.
$$

and satisfy the initial conditions $\mathbf{X}^{\epsilon}(0)=\mathbf{x}, \mathbf{P}^{\epsilon}(0)=\mathbf{p}, Q^{\epsilon}(0)=0$.

These characteristic ODEs coincide with the type of ODEs that we study in Appendix A in the particular case where $m=d$ and $l=d+1$. As a consequence, for a 
sufficiently smooth function $W_{L}$, for instance $W_{L} \in C_{b}^{2}\left(\mathbb{R}_{\mathbf{x}}^{d} \times \mathbb{R}_{\mathbf{p}}^{d}\right)$, from Theorem A.1, we get that $E\left\{W^{\epsilon}(\mathbf{x}, 0, \mathbf{p})\right\}$ converges pointwise to $W^{0}(\mathbf{x}, 0, \mathbf{p})$ as $\epsilon \rightarrow 0$, where the function $W^{0}(\mathbf{x}, z, \mathbf{p} ; k)$ satisfies in the weak sense the equation

$$
k \frac{\partial W}{\partial z}+\mathbf{p} \cdot \nabla_{\mathbf{x}} W+\frac{k^{3}}{2} \mathcal{L}_{\mathbf{p}}^{a} W=0
$$

with final condition

$$
W(\mathbf{x}, z=L, \mathbf{p} ; k)=W_{L}(\mathbf{x}, \mathbf{p}) .
$$

The differential operator $\mathcal{L}_{\mathbf{p}}^{a}$ is defined as

$$
\mathcal{L}_{\mathbf{p}}^{a} W= \begin{cases}\nabla_{\mathbf{p}} \cdot\left(D(\mathbf{0}) \nabla_{\mathbf{p}} W\right)-A(\mathbf{0}) W, & \text { if } 0<a<1, \\ \nabla_{\mathbf{p}} \cdot\left(D\left(\frac{\mathbf{p}}{k}\right) \nabla_{\mathbf{p}} W\right)-A\left(\frac{\mathbf{p}}{k}\right) W, & \text { if } a=1,\end{cases}
$$

where the positive-definite diffusion matrix $D(\tilde{\mathbf{p}})$ has elements

$$
D_{i j}(\tilde{\mathbf{p}})=\frac{1}{(2 \pi)^{d}} \int_{\mathbb{R}^{d}} \hat{R}_{\mu}(\mathbf{q}, \tilde{\mathbf{p}} \cdot \mathbf{q}) q_{i} q_{j} d \mathbf{q}, \quad i, j=1, \ldots, d
$$

and the attenuation coefficient $A(\tilde{\mathbf{p}})$ is given by

$$
A(\tilde{\mathbf{p}})=\frac{1}{(2 \pi)^{d}} \int_{\mathbb{R}^{d}} \hat{R}_{\eta}(\mathbf{q}, \tilde{\mathbf{p}} \cdot \mathbf{q}) d \mathbf{q} \geq 0 .
$$

From this observation after a simple application of the dominated convergence theorem we get the following result.

TheOREM 3.2. Assume that $k \neq 0$ and the isotropic random vector field $\left(\nabla_{\perp} \mu(\cdot), \eta(\cdot)\right)^{t}$ satisfies conditions C.1-C.5 of Appendix A (page 349) with $m=d$ and $l=d+1$.

Let the function $W_{L}(\mathbf{x}, \mathbf{p})=\hat{f}(\mathbf{p}) \chi^{2}(\mathbf{x}) \in C_{b}^{2}\left(\mathbb{R}_{\mathbf{x}}^{d} \times \mathbb{R}_{\mathbf{p}}^{d}\right) \cap L^{2}\left(\mathbb{R}_{\mathbf{x}}^{d} \times \mathbb{R}_{\mathbf{p}}^{d}\right)$. Then, for every fixed $\mathbf{x}$, the averaged Wigner transform $E\left\{W^{\epsilon}(\mathbf{x}, z=0, \mathbf{p} ; k)\right\}$ converges weakly in $L^{2}\left(\mathbb{R}_{\mathbf{p}}^{d}\right)$, as $\epsilon \rightarrow 0$, to the function $W^{0}(\mathbf{x}, z=0, \mathbf{p} ; k)$ that solves $(3.12)-(3.13)$.

In the general case, when we consider a non-smooth function $W_{L}$, the results of Appendix A do not apply directly. However, a simple approximation argument allows us to obtain a weaker version of the previous theorem.

TheOREM 3.3. Assume that $k \neq 0$ and the isotropic random vector field $\left(\nabla_{\perp} \mu(\cdot), \eta(\cdot)\right)^{t}$ satisfies conditions C.1-C.5 of Appendix A (page 349) with $m=d$ and $l=d+1$.

Let the function $W_{L}(\mathbf{x}, \mathbf{p})=\hat{f}(\mathbf{p}) \chi^{2}(\mathbf{x}) \in L^{2}\left(\mathbb{R}_{\mathbf{x}}^{d} \times \mathbb{R}_{\mathbf{p}}^{d}\right)$. Then the averaged Wigner transform $E\left\{W^{\epsilon}(\mathbf{x}, z=0, \mathbf{p} ; k)\right\}$ converges weakly in $L^{2}\left(\mathbb{R}_{\mathbf{x}}^{d} \times \mathbb{R}_{\mathbf{p}}^{d}\right)$, as $\epsilon \rightarrow 0$, to the function $W^{0}(\mathbf{x}, z=0, \mathbf{p} ; k)$ that solves $(3.12)-(3.13)$.

Proof. It is enough to prove that for any $\lambda \in C_{0}^{\infty}\left(\mathbb{R}_{\mathbf{x}}^{d} \times \mathbb{R}_{\mathbf{p}}^{d}\right)$

$$
\lim _{\epsilon \rightarrow 0} E\left\{\left\langle W^{\epsilon}(z=0, \cdot), \lambda(\cdot)\right\rangle\right\}=\left\langle W^{0}(z=0, \cdot), \lambda(\cdot)\right\rangle,
$$

where $\langle\cdot, \cdot\rangle$ represents the inner product in $L^{2}\left(\mathbb{R}_{\mathbf{x}}^{d} \times \mathbb{R}_{\mathbf{p}}^{d}\right)$.

We first consider the case of smooth data $W_{1} \in C_{0}^{\infty}\left(\mathbb{R}_{\mathbf{x}}^{d} \times \mathbb{R}_{\mathbf{p}}^{d}\right)$. Let $W_{1}^{\epsilon}(\mathbf{x}, z, \mathbf{p})$ and $W_{1}^{0}(\mathbf{x}, z, \mathbf{p})$ be the solutions of Equations (3.5) and (3.12), respectively, with 
final conditions $W_{1}^{\epsilon}(z=L, \cdot)=W_{1}^{0}(z=L, \cdot)=W_{1}(\cdot)$. Then from Theorem A.1, after applying the dominated convergence theorem, one gets that

$$
\lim _{\epsilon \rightarrow 0} E\left\{\left\langle W_{1}^{\epsilon}(z=0, \cdot), \lambda(\cdot)\right\rangle\right\}=\left\langle W_{1}^{0}(z=0, \cdot), \lambda(\cdot)\right\rangle .
$$

Next, we consider the general case. Let $\delta>0$, and choose $W_{1} \in C_{0}^{\infty}\left(\mathbb{R}_{\mathbf{x}}^{d} \times \mathbb{R}_{\mathbf{p}}^{d}\right)$ such that $\left\|W_{L}-W_{1}\right\|_{L^{2}}<\frac{\delta}{3\|\lambda\|_{L^{2}}}$. We have that

$$
\begin{aligned}
\Delta_{\lambda}^{\epsilon} W= & \left|E\left\{\left\langle W^{\epsilon}(0, \cdot), \lambda(\cdot)\right\rangle\right\}-\left\langle W^{0}(\cdot), \lambda(\cdot)\right\rangle\right| \\
\leq & \left|E\left\{\left\langle W^{\epsilon}(0, \cdot)-W_{1}^{\epsilon}(0, \cdot), \lambda(\cdot)\right\rangle\right\}\right|+\left|E\left\{\left\langle W_{1}^{\epsilon}(0, \cdot)-W_{1}^{0}(0, \cdot), \lambda(\cdot)\right\rangle\right\}\right| \\
& +\left|E\left\{\left\langle W_{1}^{0}(0, \cdot)-W(0, \cdot), \lambda(\cdot)\right\rangle\right\}\right| .
\end{aligned}
$$

Observe that since Equation (3.5) preserves the $L^{2}$-norm, we have that

$$
\left|E\left\{\left\langle W^{\epsilon}(0, \cdot)-W_{1}^{\epsilon}(0, \cdot), \lambda(\cdot)\right\rangle\right\}\right| \leq\left\|W_{L}-W_{1}\right\|_{L^{2}}\|\lambda\|_{L^{2}}<\frac{\delta}{3} .
$$

Moreover, the $L^{2}$-norm of a solution of Equation (3.12) is a non-decreasing function of $z$, hence

$$
\left|E\left\{\left\langle W_{1}^{0}(0, \cdot)-W(0, \cdot), \lambda(\cdot)\right\rangle\right\}\right| \leq\left\|W_{L}-W_{1}\right\|_{L^{2}}\|\lambda\|_{L^{2}}<\frac{\delta}{3} .
$$

Finally, from the first part of this proof we know that $E\left\{\left\langle W_{1}^{\epsilon}(0, \cdot)-\right.\right.$ $\left.\left.W_{1}^{0}(0, \cdot), \lambda(\cdot)\right\rangle\right\} \rightarrow 0$ as $\epsilon \rightarrow 0$. Thus, there is $\epsilon^{\prime}>0$ such that for any $\epsilon<\epsilon^{\prime}$,

$$
\left|E\left\{\left\langle W_{1}^{\epsilon}(0, \cdot)-W_{1}^{0}(0, \cdot), \lambda(\cdot)\right\rangle\right\}\right|<\frac{\delta}{3} .
$$

Consequently, from estimates (3.16)-(3.19), one gets that for any $\epsilon<\epsilon^{\prime}, \Delta_{\lambda}^{\epsilon} W<\delta$, and (3.15) follows.

3.3.2. Statistical stability in the diffusion limit. To complete the characterization of the asymptotics, as $\epsilon \rightarrow 0$, for the geometrical optics Wigner transform we establish in this section the celebrated self-averaging property.

For a sufficiently smooth function $W_{L}$, for instance $W_{L} \in C_{b}^{2}\left(\mathbb{R}_{\mathbf{x}}^{d} \times \mathbb{R}_{\mathbf{p}}^{d}\right)$, from Theorem A.4, we know that the limiting averaged Wigner transform decorrelates. More exactly, we have the pointwise convergence

$$
E\left\{W^{\epsilon}\left(\mathbf{x}_{1}, 0, \mathbf{p}_{1}\right) \overline{W^{\epsilon}\left(\mathbf{x}_{2}, 0, \mathbf{p}_{2}\right)}\right\} \rightarrow W^{0}\left(\mathbf{x}_{1}, 0, \mathbf{p}_{1}\right) \overline{W^{0}\left(\mathbf{x}_{2}, 0, \mathbf{p}_{2}\right)}, \quad \text { for } \mathbf{p}_{1} \neq \mathbf{p}_{2}
$$

as $\epsilon \rightarrow 0$. Using this result, we can characterize the limiting second order moments and establish that the variance of the limiting Wigner transform equals zero. This means that the limiting Wigner transform is statistically stable. More specifically, we have the following result.

THEOREM 3.4. Assume that $d \geq 2, k \neq 0$ and the isotropic random vector field $\left(\nabla_{\perp} \mu(\cdot), \eta(\cdot)\right)^{t}$ satisfies conditions C.1-C.5 of Appendix $A$ (page 349) with $m=d$ and $l=d+1$.

Let the function $W_{L}(\mathbf{x}, \mathbf{p})=\hat{f}(\mathbf{p}) \chi^{2}(\mathbf{x}) \in L^{2}\left(\mathbb{R}_{\mathbf{x}}^{d} \times \mathbb{R}_{\mathbf{p}}^{d}\right)$. Then the Wigner transform $W^{\epsilon}(\mathbf{x}, z=0, \mathbf{p} ; k)$ given by Equations (3.5)-(3.6) converges in probability and weakly in $L^{2}\left(\mathbb{R}_{\mathbf{x}}^{d} \times \mathbb{R}_{\mathbf{p}}^{d}\right)$, as $\epsilon \rightarrow 0$, to the function $W^{0}(\mathbf{x}, z=0, \mathbf{p} ; k)$ that solves (3.12)(3.13). More precisely, for any fixed test function $\lambda \in L^{2}\left(\mathbb{R}_{\mathbf{x}}^{d} \times \mathbb{R}_{\mathbf{p}}^{d}\right)$ the random variable $\left\langle W^{\epsilon}(0, \cdot), \lambda(\cdot)\right\rangle$ converges in probability to $\left\langle W^{0}(0, \cdot), \lambda(\cdot)\right\rangle$ as $\epsilon \rightarrow 0$. 
Furthermore, when $W_{L}(\mathbf{x}, \mathbf{p}) \in C_{b}^{2}\left(\mathbb{R}_{\mathbf{x}}^{d} \times \mathbb{R}_{\mathbf{p}}^{d}\right) \cap L^{2}\left(\mathbb{R}_{\mathbf{x}}^{d} \times \mathbb{R}_{\mathbf{p}}^{d}\right)$ the Wigner transform $W^{\epsilon}(\mathbf{x}, z=0, \mathbf{p} ; k)$ converges in probability and weakly in $L^{2}\left(\mathbb{R}_{\mathbf{p}}^{d}\right)$, for every fixed $\mathbf{x}$.

The proof is similar to that of Theorems 3.2 and 3.3.

Proof. It is enough to establish that for any $\lambda \in L^{2}\left(\mathbb{R}_{\mathbf{x}}^{d} \times \mathbb{R}_{\mathbf{p}}^{d}\right)$ we have that

$$
\lim _{\epsilon \rightarrow 0} E\left\{\left|\left\langle W^{\epsilon}(0, \cdot), \lambda(\cdot)\right\rangle\right|^{2}\right\}=\left|\left\langle W^{0}(0, \cdot), \lambda(\cdot)\right\rangle\right|^{2} .
$$

Note that

$$
\begin{aligned}
& \left|\left\langle W^{\epsilon}(0, \cdot), \lambda(\cdot)\right\rangle\right|^{2}=\left\langle\left(W^{\epsilon} \otimes \overline{W^{\epsilon}}\right)(0, \cdot),(\lambda \otimes \bar{\lambda})(\cdot)\right\rangle, \\
& \left|\left\langle W^{\epsilon}(0, \cdot), \lambda(\cdot)\right\rangle\right|^{2}=\left\langle\left(W^{0} \otimes \overline{W^{0}}\right)(0, \cdot),(\lambda \otimes \bar{\lambda})(\cdot)\right\rangle,
\end{aligned}
$$

where

$$
\left(V_{1} \otimes V_{2}\right)\left(\mathbf{x}_{1}, \mathbf{x}_{2}, \mathbf{p}_{1}, \mathbf{p}_{2}\right)=V_{1}\left(\mathbf{x}_{1}, \mathbf{p}_{1}\right) V_{2}\left(\mathbf{x}_{2}, \mathbf{p}_{2}\right)
$$

represents the tensor product of functions and $\langle\cdot, \cdot\rangle$ stands for the inner product in $L^{2}\left(\mathbb{R}_{\mathbf{x}_{1}}^{d} \times \mathbb{R}_{\mathbf{p}_{1}}^{d} \times \mathbb{R}_{\mathbf{x}_{2}}^{d} \times \mathbb{R}_{\mathbf{p}_{2}}^{d}\right)$.

Let $\mathcal{T}_{\mathbf{x}, \mathbf{p}}^{\epsilon}, \tilde{\mathcal{T}}_{\mathbf{x}, \mathbf{p}}^{\epsilon}$ represent the transport operator appearing in Equation (3.5) and its complex conjugate, respectively, and $\mathcal{D}_{\mathbf{x}, \mathbf{p}}$ the transport-diffusion operator appearing in Equation (3.12). We have that $W^{\epsilon} \otimes W^{\epsilon}$ satisfies the equation

$$
\left(k \frac{\partial}{\partial z}+\mathcal{T}_{\mathbf{x}_{1}, \mathbf{p}_{1}}^{\epsilon}+\tilde{\mathcal{T}}_{\mathbf{x}_{2}, \mathbf{p}_{2}}^{\epsilon}\right) V=0
$$

with the final condition $V(z=L, \cdot)=\left(W_{L} \otimes \overline{W_{L}}\right)(\cdot)$. Additionally, $W^{0} \otimes \overline{W^{0}}$ solves equation

$$
\left(k \frac{\partial}{\partial z}+\mathcal{D}_{\mathbf{x}_{1}, \mathbf{p}_{1}}+\mathcal{D}_{\mathbf{x}_{2}, \mathbf{p}_{2}}\right) \bar{V}=0
$$

with the additional condition $\bar{V}(z=L, \cdot)=\left(W_{L} \otimes \overline{W_{L}}\right)(\cdot)$.

On one hand, from Theorem A.4 it follows that for the solution $V^{\epsilon}(z, \cdot)$ of Equation $(3.21)$ with a sufficiently smooth final condition $\left(W_{1} \otimes \overline{W_{1}}\right)(\cdot)$, we have that $E\left\{V^{\epsilon}(0, \cdot)\right\}$ converges pointwise to $\bar{V}(0, \cdot)$, which solves Equation $(3.22)$ with the final condition $\left(W_{1} \otimes \overline{W_{1}}\right)(\cdot)$.

On the other hand, it is not difficult to prove that Equation (3.21) preserves the $L^{2}$-norm and that the solutions of (3.22) have an $L^{2}$-norm non-increasing with $z$.

Using these facts, the proof can be completed in the same way as in Theorem 3.3. (

3.4. Statistical stability of the back-propagated wave. From the results obtained in the previous section, one can establish the statistical stabilization (or selfaveraging) of the limiting time-reversed back-propagated wave. This property can be stated as follows:

$$
\begin{aligned}
\phi_{\theta}^{B}\left(\boldsymbol{\xi}, \mathbf{x}_{0} ; k\right) & \rightarrow \bar{\phi}^{B}\left(\boldsymbol{\xi}, \mathbf{x}_{0} ; k\right) \quad \text { as }(\theta \ll \epsilon) \rightarrow 0 \text { for } k \neq 0, \\
u_{\theta}^{B}\left(\boldsymbol{\xi}, \mathbf{x}_{0}, t\right) & \rightarrow \bar{u}^{B}\left(\boldsymbol{\xi}, \mathbf{x}_{0}, t\right) \quad \text { as }(\theta \ll \epsilon) \rightarrow 0,
\end{aligned}
$$

where the convergence is considered in probability. 
Moreover, from the previous section, it is apparent that the convergence of the (complex) wave amplitude should be in a weak sense, since we need to average with respect to $\mathbf{x}_{0}$. Concerning the back-propagated wave, we have a similar situation with respect to $\mathbf{x}_{0}$, while in the time domain we still can have a stronger convergence. More specifically, we say that a sequence of functions $f_{n} \in L^{2}\left(\mathbb{R}_{\mathrm{x}}^{d} \times \mathbb{R}_{t}\right)$ converges semiweakly to $f$ if for every $\lambda \in L^{2}\left(\mathbb{R}_{\mathbf{x}}^{d}\right)$

$$
\left\|\left\langle f_{n}-f, \lambda\right\rangle\right\|_{L^{2}\left(\mathbb{R}_{t}\right)} \rightarrow 0 .
$$

THEOREM 3.5. Let $d \geq 2$ and assume that the isotropic random vector field $\left(\nabla_{\perp} \mu(\cdot), \eta(\cdot)\right)^{t}$ satisfies conditions C.1-C.5 of Appendix A (page 349) with $m=d$ and $l=d+1$. For each $\boldsymbol{\xi} \in \mathbb{R}^{d}$ and almost every $k \neq 0$, the amplitude of the backpropagated wave $\phi_{\theta}^{B}\left(\boldsymbol{\xi}, \mathbf{x}_{0} ; k\right)$ given by $(2.17)$ converges in probability and weakly in $L^{2}\left(\mathbb{R}_{\mathbf{x}_{0}}^{d}\right)$, as $(\epsilon \ll \theta) \rightarrow 0$, to the deterministic function $\bar{\phi}^{B}\left(\boldsymbol{\xi}, \mathbf{x}_{0} ; k\right)$ given by (3.2), where $W^{0}\left(\mathbf{x}_{0}, 0, \mathbf{p} ; k\right)$ is the solution of Equation (3.12) with final condition (3.13).

Additionally, the back-propagated wave $u_{\theta}^{B}\left(\boldsymbol{\xi}, \mathbf{x}_{0}, t\right)$ converges in probability and semi-weakly in $L^{2}\left(\mathbb{R}_{\mathbf{x}_{0}}^{d} \times \mathbb{R}_{t}\right)$ to the deterministic wave $\bar{u}^{B}\left(\boldsymbol{\xi}, \mathbf{x}_{0}, t\right)$ given by Equation (3.1).

Proof. Using Theorem 3.1, the convergence of the amplitude of the backpropagated wave follows by applying Theorem 3.4 with test functions of the form $\lambda_{1}(\mathbf{x}, \mathbf{p} ; k)=e^{i \mathbf{p} \boldsymbol{\xi}} \lambda(\mathbf{x}) \tilde{\phi}_{0}(\mathbf{p} ; k)$.

For the back-propagated wave, notice first that it is enough to establish that

$$
I=E\left\{\int\left|\left\langle\phi_{\epsilon}^{B}(\boldsymbol{\xi}, \cdot ; k)-\bar{\phi}^{B}(\boldsymbol{\xi}, \cdot ; k), \lambda(\cdot)\right\rangle\right|^{2} d k\right\}
$$

converges to zero. Rewriting $I$ as

$$
I=\int E\left\{\left|\left\langle W^{\epsilon}(0, \cdot ; k)-W^{0}(0, \cdot ; k), \lambda_{1}(\cdot ; k)\right\rangle\right|^{2}\right\} d k
$$

we know from Theorem 3.4 that the integrand function above converges to zero for almost every $k \neq 0$, and since $W^{\epsilon}$ and $W^{0}$ solve Equations (3.5)-(3.6) and (3.12)(3.13), respectively, it is bounded by the integrable function $C_{1}\|\lambda\|_{L^{2}}^{2}\left\|\tilde{\phi}_{0}(\cdot ; k)\right\|_{L^{2}\left(\mathbb{R}_{\mathbf{p}}^{d}\right)}^{2}$. Finally, using the dominated convergence theorem it follows that $I \rightarrow 0$.

\section{Time-reversal super-focusing and stability}

The results presented above give us a complete asymptotic characterization of the back-propagated wave. It is remarkable that, despite the perturbations, the timereversed back-propagated wave remains statistically stable, and the refocused spot has a better resolution than the one corresponding to the homogeneous medium; we describe this phenomenon next.

4.1. Perturbation effects on the refocused wave. First, note that when there is no perturbation, the unperturbed limiting Wigner transform $W_{\text {unp }}^{0}$ satisfies the equation

$$
k \frac{\partial W}{\partial z}+\mathbf{p} \cdot \nabla_{\mathbf{x}} W+\frac{k^{3}}{2} \overline{\mathcal{L}}_{\mathbf{p}}^{a} W=0
$$

with final condition

$$
W(\mathbf{x}, z=L, \mathbf{p} ; k)=W_{L}(\mathbf{x}, \mathbf{p} ; k),
$$


where

$$
\overline{\mathcal{L}}_{\mathbf{p}}^{a} W= \begin{cases}\nabla_{\mathbf{p}} \cdot\left(D(\mathbf{0}) \nabla_{\mathbf{p}} W\right) & \text { if } 0<a<1, \\ \nabla_{\mathbf{p}} \cdot\left(D\left(\frac{\mathbf{p}}{k}\right) \nabla_{\mathbf{p}} W\right) & \text { if } a=1\end{cases}
$$

The associated limiting unperturbed (complex) wave amplitude and back-propagated wave are then given by

$$
\bar{\phi}_{\text {unp }}^{B}\left(\boldsymbol{\xi}, \mathbf{x}_{0} ; k\right)=\int_{\mathbb{R}^{d}} e^{-i \mathbf{p} \cdot \boldsymbol{\xi}} W_{\text {unp }}^{0}\left(\mathbf{x}_{0}, 0, \mathbf{p} ; k\right) \tilde{\phi}_{0}(\mathbf{p} ; k) d \mathbf{p}
$$

and

$$
\bar{u}_{\text {unp }}^{B}\left(\boldsymbol{\xi}, \mathbf{x}_{0}, t\right)=k_{0} \int e^{i k k_{0} c_{0} t} \bar{\phi}_{\text {unp }}^{B}\left(\boldsymbol{\xi}, \mathbf{x}_{0} ; k\right) d k .
$$

This is in complete agreement with the analysis presented in [30], since when $a=1$ we recover the advection-diffusion equation derived there.

Therefore, the effect of perturbations corresponds to the additional reaction term in (3.12) characterized by the attenuation coefficient $A(\cdot)$. Furthermore, in the case where $0<a<1, A(\cdot) \equiv A_{0}=$ const. Consequently, one can readily quantify the effect of perturbations since the limiting Wigner distributions satisfy

$$
W^{0}(\mathbf{x}, 0, \mathbf{p} ; k)=W_{\text {unp }}^{0}(\mathbf{x}, 0, \mathbf{p} ; k) e^{-\frac{k^{2}}{2} A_{0} L} .
$$

For the limiting (complex) wave amplitude and back-propagated wave we then get

$$
\begin{aligned}
\bar{\phi}^{B}\left(\boldsymbol{\xi}, \mathbf{x}_{0} ; k\right) & =\bar{\phi}_{\text {unp }}^{B}\left(\boldsymbol{\xi}, \mathbf{x}_{0} ; k\right) e^{-\frac{k^{2}}{2} A_{0} L}, \\
\bar{u}^{B}\left(\boldsymbol{\xi}, \mathbf{x}_{0}, t\right) & =\left(G(\cdot) \star \bar{u}_{\text {unp }}^{B}\left(\boldsymbol{\xi}, \mathbf{x}_{0}, \cdot\right)\right)(t),
\end{aligned}
$$

where $\star$ stands for convolution in time and $G$ is given by the Gaussian kernel

$$
G(t)=\frac{1}{\sqrt{2 \pi} \sigma_{G}} e^{-\frac{t^{2}}{2 \sigma_{G}^{2}}}
$$

where $\sigma_{G}=\frac{\sqrt{A_{0} L}}{k_{0} c_{0}}$. Note that $A_{0}$ represents a statistical measure of the perturbation's intensity. We have

$$
A_{0}=\int R_{\eta}(\mathbf{0}, z) d z
$$

so that this parameter is the longitudinal correlation length in the case with anisotropic medium fluctuations.

It is remarkable that the perturbations produce an exponential attenuation of the wave amplitude when compared with its counterpart from the unperturbed case. At a fixed frequency the perturbations result in a rescaling factor that does not depend on the space variable and does not affect the space resolution of the wave amplitude. Thus, the perturbations produce a time domain smearing effect on the backpropagated pulse when compared to the time reversal in an unperturbed medium. In particular, if the initial pulse is a Gaussian time-pulse with carrier frequency $\omega_{0}=k_{0} c_{0}$ and width $\sigma_{t}$ then the back-propagated signal in the perturbed medium coincides with 
the recompressed wave in the unperturbed medium generated by an effective initial pulse. This effective initial pulse is a Gaussian with carrier frequency $\tilde{\omega}_{0}=\frac{\omega_{0}}{\gamma}$ and pulse width $\tilde{\sigma}_{t}=\sigma_{t} \gamma$, rescaled by the factor $\gamma \exp \left(-A_{0} L / 2 \gamma\right)$, where $\gamma=1+\sigma_{G}^{2} / \sigma_{t}^{2}$. This is a manifestation of the attenuation of the back-propagated wave, caused by perturbations that have been observed in physical experiments [34] and is also valid in other asymptotic regimes [5, 7].

Concerning space resolution, it is apparent from Equations (4.2)-(4.3) that perturbations do not affect the space super-resolution of the back-propagated wave when compared with time reversal in an unperturbed medium. This means that the wave pulse $\bar{u}_{0}^{B}$ has a tighter support in the case of a random medium than in the absence of medium fluctuations and multipathing, even though the back-propagation takes place in a slightly modified random medium. In other words, relatively weak perturbations do not eliminate the super-focusing of the recompressed wave. Furthermore, the recompressed wave is statistically stable, i.e., its shape does not depend on the medium and perturbation realizations.

Super-focusing in the case without medium perturbations is discussed, for instance, in $[11,30,31]$. In particular, in [30] the asymptotic regime corresponding to the case where $a=1$ is treated. Therefore, their observations concerning the effective aperture of the TRM are also valid for our case. Moreover, when $a<1$ and the diffusion matrix is $D_{i j}=D \delta_{i j}$ with a constant diffusion coefficient $D$ we have that the effective aperture of the TRM is given by $a_{\mathrm{TRM}}^{e f f}=\sqrt{a_{\mathrm{TRM}}^{2}+\frac{D L^{3}}{3}}$, where $a_{\mathrm{TRM}}$ represents the actual aperture of the TRM and $L$ the distance from the source to the TRM.

4.2. Numerical results. The conclusions in the previous section are obtained under the condition $a<1$. The results of the numerical simulations, carried out for the case where $a=1$, show that this restriction is not fundamental. More specifically, we conclude in this situation and in the presence of perturbations, that the recompressed wave remains statistically stable (i.e the back-propagated wave is self-averaging) and its space super-resolution is not affected, but the wave amplitude experience a frequency-dependent attenuation.

All the numerical examples correspond to the $2 D$ setting. We set the propagation velocity $c_{0}=1$ and consider the central wave length $\lambda_{0}=2 \pi / k_{0}=2 \pi / \omega_{0}$, where $k_{0}$ and $\omega_{0}$ are the central wave number and frequency, respectively. Consider the following values for the characteristic lengths involved: let the TRM aperture be $a_{\mathrm{TRM}}=250 \lambda_{0}$, the distance from the TRM to the source be $L=5000 \lambda_{0}$, and let the transversal and longitudinal correlation lengths of the medium fluctuations be $l_{x}=5 \lambda_{0}$ and $l_{z}=100 \lambda_{0}$, respectively. Furthermore, we ensure a high frequency regime by taking $\omega_{0}=k_{0}=2 \pi$. Note that this set of values satisfies the restrictions corresponding to the asymptotic regime introduced in Section 2.1 in the case when $a=1$. The random fluctuations are stationary centered Gaussian random fields with an exponential autocorrelation function; they are constructed spectrally. The maximum contrast is $10 \%$ for the forward medium, and the incremented independent perturbations represent $5 \%$ of the backward medium. As initial source we take $\phi_{0}\left(\mathbf{x} ; k_{0}\right)=\exp \left\{-\frac{|\mathbf{x}|^{2}}{2 \sigma_{s}^{2}}\right\}$ with $\sigma_{s}=3 \lambda_{0}$.

In the numerical simulations the Schrödinger equation is solved using a FD code; to model the infinite medium a perfectly matched layer that allows plane wave absorption through the (computational) lateral boundaries is introduced. The computational domain has a $4 a_{\mathrm{TRM}}$ width and its discretization uses $\Delta x=0.25 \lambda_{0}$ and $\Delta z=0.5 \lambda_{0}$.

In Figure 4.1 we show the super-resolution of the back-propagated wave. First, 
we compare the recompressed wave amplitude for the homogeneous medium and an individual realization of the unperturbed random medium. The left plot shows that a sharper recompression is obtained in the latter situation. Thus, the presence of inhomogeneities and the multipathing enhances the resolution of the recompressed wave. In the right plot we compare the recompressed wave amplitude for individual realizations of the unperturbed and perturbed random media. The resolution in both cases is similar, while the maximum amplitude is higher for the unperturbed case. As predicted, the perturbations do not affect the spatial resolution of the back-propagated wave, but attenuate its amplitude.
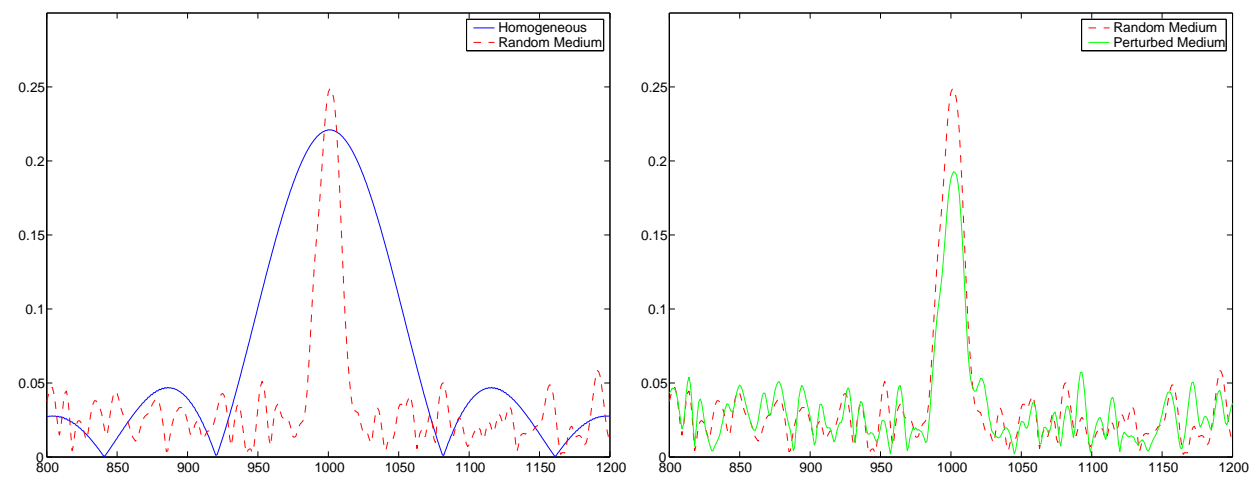

FIG. 4.1. Super-focusing of the back-propagated wave. The absolute value of the recompressed wave amplitude at the central wave number $k_{0}=2 \pi$ is shown. Left plot: Homogeneous vs. (unperturbed) random media; the former is shown as a solid line and the latter with a dashed line. Right plot: Unperturbed vs. perturbed random media; the former is shown as a dashed line and the latter with a solid one.

In Figure 4.2 (left) we illustrate the statistical stabilization (or self-averaging) of the recompressed wave. The results from a set of 10 time reversal experiments are plotted. It is remarkable that for all medium realizations the resolution of the back-propagated wave are quite similar. It is also remarkable that near the center the recompressed waves for different realizations vary less than at the sides. Although we only show the results of 10 realizations, we remark that other numerical simulations exhibit analogous results.

In the right plot of Figure 4.2, we illustrate the attenuation effect of the perturbations on the recompressed wave amplitude for different wave numbers. We present the resulting maximum wave amplitude ratio of the perturbed to unperturbed random media, corresponding to 26 values of the wave number $k$ uniformly located in the interval $[3 \pi / 2,4 \pi]$. It is apparent from this plot that the wave amplitude decays as frequency increases, leading to the attenuation of the back-propagated pulse.

4.3. Lateral diversity and stability. Generally speaking, in the parabolic regime, the statistical stability of the back-propagated wave comes from the decorrelation of the Wigner transform in the frequency domain or in phase space. Then, integration in the frequency domain or in phase space averages out the random variations of the Wigner transform. It is worth noticing that in the situation analyzed in this work, as well as in other high-frequency regimes (see for instance $[11,31]$ ), the underlying physical mechanism leading to the mentioned decorrelation is diffusion in phase space. In fact, the high-frequency asymptotics of the Wigner transform 

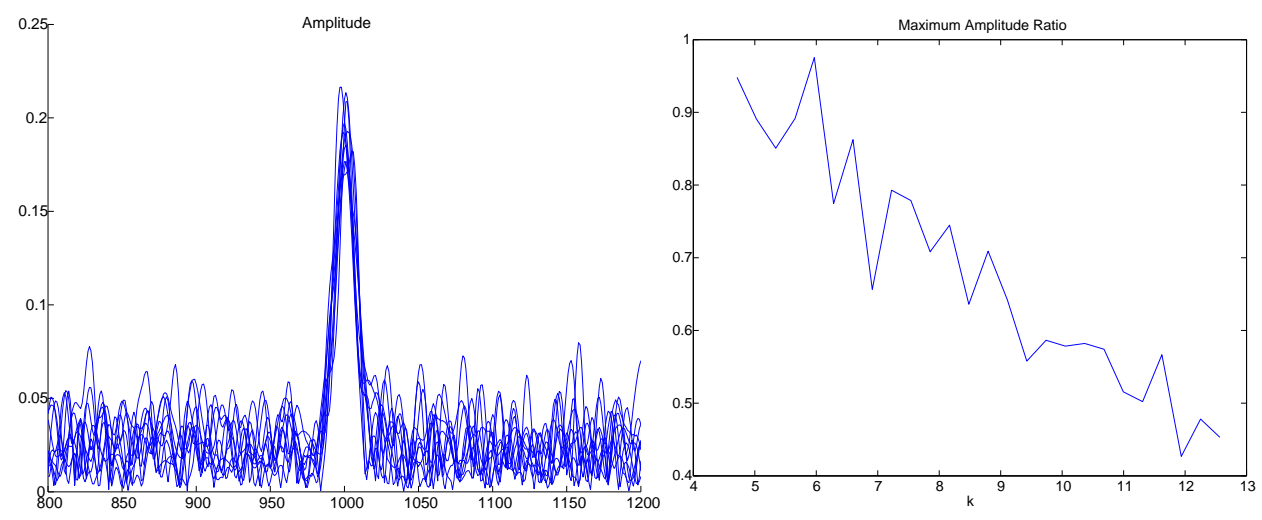

FIG. 4.2. Left plot: Self-averaging of the back-propagated wave in a perturbed random medium. We show the absolute value of the recompressed wave amplitude at the central wave number $k_{0}=2 \pi$ for 10 realizations of the (perturbed) random medium. Right plot: Wave amplitude Ratio of the perturbed to unperturbed random media at the central position for different wave numbers in the interval

are related to a Liouville equation. Under appropriate conditions, the trajectories associated with the Liouville equation have a diffusive behavior, and two trajectories starting at different points of the phase space remain well-separated. Finally, if the random fluctuations of the medium at the points of the trajectories decorrelates sufficiently fast, then the decorrelation of the corresponding solutions of the Liouville equation follows.

A fast decorrelation of the medium fluctuations is enforced by considering a large lateral diversity, i.e., the typical width of the beam is large compared to the corresponding correlation length. The self-averaging, in time reversal without perturbations that results from lateral diversity is analyzed in [31, 32].

For time reversal without perturbations, the results obtained in [27] lead to the conclusion that in the high-frequency regime lateral diversity is a necessary condition for statistical stability. Indeed, there it is shown that in the regime corresponding to $a=0$ in the scaling (2.6) the Wigner transform does not decorrelate. The results in the present work and in $[31,32]$ show that as soon as some lateral diversity is present, a statistically stable recompressed wave is obtained. Moreover, we proved here that relatively weak perturbations do not affect the self-averaging.

4.4. Concluding remarks. We have analyzed and explained the effect of medium perturbations during a time reversal experiment. Our analysis was carried out for a high frequency regime where the TRM size is much smaller than the propagation distance. The medium is anisotropic and its longitudinal and transversal correlation lengths are much smaller than the propagation distance and the TRM size, respectively, and the first ratio is smaller than or comparable to the second. Furthermore, the fluctuations of the refractive index are weak and the perturbations are relatively small, and we considered an initial pulse generated by a localized source.

We proved that perturbations do not affect the main properties of the re-compressed wave, namely, super-resolution and statistical stability, but produce a pulse smeared in time. Moreover, the influence of the perturbations can be quantified through the statistical properties of the medium. 
Acknowledgement. The authors want to thank Prof. K. Huang from UCI for his help with the numerical simulations.

Appendix A. Diffusion limit of the characteristic ODE .

In this appendix, we study the weak convergence of stochastic processes associated with a system of ODEs with a special form. These auxiliary results are used in the main part of the paper to study the characteristic ODEs (3.11). Actually, we will study a system of ODEs slightly more general than these equations. Our analysis follows the same lines as in $[27,28]$.

This appendix is organized as follows. In Section A.1 we present the conditions satisfied by the random field associated with the right-hand side of the system of ODEs. In Section A.2, we study the one-particle problem and characterize the weak limit of the solution to the ODEs. Section A.3 is concerned with the two-particle problem; that is, we analyze the weak convergence of the joint solution to the ODEs that starts at two different points.

A.1. Introduction. Let $(\Omega, \mathcal{F}, P)$ be a probability space and the vector field $F: \mathbb{R}^{m+1} \times \Omega \rightarrow \mathbb{R}^{l}$ be jointly measurable with respect to $\mathcal{B}^{m+1} \times \mathcal{F}$, where $\mathcal{B}^{m+1}$ is the $\sigma$-algebra of Borel sets in $\mathbb{R}^{m+1}$, and assume that $m \leq l$. We also assume that the random field $F(\mathbf{y})=F(\mathbf{y}, \omega)$ is stationary and sufficiently smooth with respect to $\mathbf{y}$. We use the representation $\|f\|_{\infty}=\operatorname{ess}_{-\sup _{\omega \in \Omega}}|f(\cdot, \omega)|$.

More specifically, we assume that the random field $F$ satisfies the following conditions:

(C.1) The random field $F$ is strictly stationary and has mean zero, i.e., $E\{F(\mathbf{y})\}=$ $\int_{\Omega} F(\mathbf{y}, \omega) P(d \omega)=0, \forall \mathbf{y} \in \mathbb{R}^{m+1}$

(C.2) The derivatives $\frac{\partial}{\partial \mathbf{y}^{\alpha}} F(\mathbf{y}, \omega)=\frac{\partial^{|\alpha|}}{\partial y_{0}^{\alpha_{0}} \ldots \partial y_{m}^{\alpha_{m}}} F(\mathbf{y}, \omega)$ exist for $P$ almost all $\omega$ and satisfy the continuity condition

$$
\lim _{\substack{\mathbf{h} \in \mathbb{R}^{m} \\|\mathbf{h}| \rightarrow 0}}\left\|\frac{\partial}{\partial \mathbf{y}^{\alpha}} F(s, \mathbf{h})-\frac{\partial}{\partial \mathbf{y}^{\alpha}} F(s, \mathbf{0})\right\|_{\infty}=0,
$$

when the index $\alpha$ equals $\left(0, \alpha_{1}, \ldots, \alpha_{m}\right)$ and $|\alpha|=\sum_{i=0}^{m} \alpha_{i} \leq 3$.

(C.3) $F$ is exponentially $\phi$-mixing. More precisely, for $\Lambda \subset \mathbb{R}^{m+1}$ set

$$
\mathcal{G}_{\Lambda}=\sigma\{F(\mathbf{y}, \cdot): \mathbf{y} \in \Lambda\}
$$

and for $\Lambda_{j} \subset \mathbb{R}^{m+1}, j=1,2$ define

$$
\phi\left(\Lambda_{1}, \Lambda_{2}\right)=\sup _{A \in \mathcal{G}_{\Lambda_{1}, B \in \mathcal{G}_{\Lambda_{2}}}}|P(B)-P(B \mid A)| .
$$

Define the uniform mixing rate as

$$
\phi(\varrho)=\sup \left\{\phi\left(\Lambda_{1}, \Lambda_{2}\right): \Lambda_{j} \in \mathcal{B}^{m+1}, d\left(\Lambda_{1}, \Lambda_{2}\right) \geq \varrho\right\},
$$

where $d\left(\Lambda_{1}, \Lambda_{2}\right)=\inf \left\{\left|\mathbf{y}_{1}-\mathbf{y}_{2}\right|: \mathbf{y}_{i} \in \Lambda_{i}\right\}$; then there exists a constant $C_{1}>0$ such that

$$
\phi(\varrho) \leq 2 e^{-C_{1} \varrho}, \quad \varrho>0 .
$$


(C.4) We let

$$
R(\mathbf{y})=E\{F(\mathbf{y}) \otimes F(\mathbf{0})\}, \quad \mathbf{y} \in \mathbb{R}^{m+1}
$$

be the covariance matrix of the vector field $F(\cdot)$. Note that (A.3) implies that there exists a constant $C_{2}$ such that

$$
\left|\frac{\partial}{\partial \mathbf{y}^{\alpha}} R_{i j}(\mathbf{y})\right| \leq C_{2} e^{-\frac{|\mathbf{y}|_{2}}{C_{2}}} \quad \forall \mathbf{y} \in \mathbb{R}^{m+1}, \quad i, j=1, \ldots, l,
$$

where $|\mathbf{y}|_{2}$ represents the euclidean norm of the vector $\mathbf{y}$ and the index $\alpha$ equals $\left(0, \alpha_{1}, \ldots, \alpha_{m}\right)$ with $|\alpha| \leq 2$.

(C.5) (a) Assume that $R_{i j} \in C^{\infty}\left(\mathbb{R}^{m+1}\right), i, j=1, \ldots, l$. Consider the $l \times l$ matrix $A(\tilde{\mathbf{q}})$ and the $l$-dimensional vector $b(\tilde{\mathbf{q}})$ with elements

$$
A_{i j}(\tilde{\mathbf{q}})=a_{i j}(\tilde{\mathbf{q}})+a_{j i}(\tilde{\mathbf{q}}) \quad \text { and } \quad b_{i}(\tilde{\mathbf{q}})=\sum_{j=1}^{m} \frac{\partial a_{i j}}{\partial q_{j}}(\tilde{\mathbf{q}})
$$

where

$$
a_{i j}(\tilde{\mathbf{q}})=\int_{0}^{\infty} R_{i j}(s, s \tilde{\mathbf{q}}) d s .
$$

(b) Suppose that the matrix $A(\tilde{\mathbf{q}})$ is positive-definite and let $C(\tilde{\mathbf{q}})$ be its positive symmetric square root. We further assume that the $m \times m$ submatrix $\tilde{C}(\tilde{\mathbf{q}})$ with elements $\tilde{C}_{i j}(\tilde{\mathbf{q}})=C_{i j}(\tilde{\mathbf{q}}), i, j=1, \ldots, m$ is nonsingular $\forall \tilde{\mathbf{q}} \in \mathbb{R}^{m}$.

A.2. One-particle problem. For any $0<\epsilon \leq 1$ define the stochastic process $\left(\mathbf{x}^{\epsilon}(s), \mathbf{q}^{\epsilon}(s)\right)=\left(\mathbf{x}^{\epsilon}(s, \omega), \mathbf{q}^{\epsilon}(s, \omega)\right)$ with values in $\mathbb{R}^{m} \times \mathbb{R}^{l}, s \geq 0, \omega \in \Omega$ as the solution of the system of ODEs

$$
\left\{\begin{array}{l}
\frac{d \mathbf{x}^{\epsilon}}{d s}=\tilde{\mathbf{q}}^{\epsilon} \\
\frac{d \mathbf{q}^{\epsilon}}{d s}=\frac{1}{\sqrt{\epsilon}} F\left(\frac{s}{\epsilon}, \frac{\mathbf{x}^{\epsilon}}{\epsilon^{a}}\right) \\
\left(\mathbf{x}^{\epsilon}(0), \mathbf{q}^{\epsilon}(0)\right)=\left(\mathbf{x}_{0}, \mathbf{q}_{0}\right),
\end{array}\right.
$$

where $\left(\mathbf{x}_{0}, \mathbf{q}_{0}\right)$ is non-random and $\tilde{\mathbf{q}}^{\epsilon}$ represents the $\mathbb{R}^{m}$-projection of $\mathbf{q}^{\epsilon}$.

Next, we focus on the weak convergence of the stochastic process defined above as $\epsilon$ goes to zero.

Consider the diffusion operator

$$
\mathcal{L}^{a} f(\mathbf{q})= \begin{cases}\frac{1}{2} \sum_{i, j=1}^{l} A_{i j}(\tilde{\mathbf{q}}) \frac{\partial^{2} f}{\partial q_{i} \partial q_{j}}(\mathbf{q})+\sum_{i=1}^{l} b_{i}(\tilde{\mathbf{q}}) \frac{\partial f}{\partial q_{i}}(\mathbf{q}), & \text { if } a=1 \\ \frac{1}{2} \sum_{i, j=1}^{l} A_{i j}(\mathbf{0}) \frac{\partial^{2} f}{\partial q_{i} \partial q_{j}}(\mathbf{q})+\sum_{i=1}^{l} b_{i}(\mathbf{0}) \frac{\partial f}{\partial q_{i}}(\mathbf{q}), & \text { if } 0<a<1\end{cases}
$$

for all $f \in C_{b}^{2}\left(\mathbb{R}^{l}\right)$. From the conditions above follows that this operator is well-defined. Furthermore, if the random field $F(\cdot)$ is isotropic then we have that

$$
\left(\mathcal{L}^{a} f\right)(\mathbf{q})= \begin{cases}\frac{1}{2} \nabla_{\mathbf{q}} \cdot\left(A(\tilde{\mathbf{q}}) \nabla_{\mathbf{q}} f\right), & \text { if } a=1 \\ \frac{1}{2} \nabla_{\mathbf{q}} \cdot\left(A(\mathbf{0}) \nabla_{\mathbf{q}} f\right), & \text { if } 0<a<1\end{cases}
$$


and

$$
A_{i j}(\tilde{\mathbf{q}})=\int_{-\infty}^{+\infty} R_{i j}(s, s \tilde{\mathbf{q}}) d s
$$

For a positive integer $n$, let us represent by $\mathcal{C}^{n}=C\left([0, \infty) ; \mathbb{R}^{n}\right)$, the space of continuous functions from $[0, \infty)$ to $\mathbb{R}^{n}$. The following theorem is a generalization of the results in $[27,28]$.

Theorem A.1. Suppose that conditions C.1-C.3 and C.5(a) above are fulfilled. Then the stochastic process $\left(\mathbf{x}^{\epsilon}(s), \mathbf{q}^{\epsilon}(s)\right)$ converges weakly in $\mathcal{C}^{m+l}$ as $\epsilon \rightarrow 0$ to the process $(\mathbf{x}(s), \mathbf{q}(s))$ such that

$$
\mathbf{x}(s)=\mathbf{x}_{0}+\int_{0}^{s} \tilde{\mathbf{q}}\left(s^{\prime}\right) d s^{\prime}
$$

and $\mathbf{q}(s)$ is the diffusion process in $\mathcal{C}^{l}$ with generator $\mathcal{L}^{a}$ defined by (A.6) starting from $\mathbf{q}_{0}$.

The proof of the above theorem is very similar to that of Theorem 3 in [27](p. $105)$ and consequently we only highlight the main ideas. Let $R^{\epsilon}$ denote the probability measure induced by $\mathbf{q}^{\epsilon}(s)$ on $\mathcal{C}^{l}$. It is enough to establish that $R^{\epsilon}$ weakly converges to $R_{\mathbf{q}_{0}}$, the probability measure of a diffusion in $\mathcal{C}^{l}$ with generator $\mathcal{L}^{a}$ and starting from $\mathbf{q}_{0}$. The main idea of the proof is to study a truncated process whose dynamic up to a certain stopping time coincides with that of the original process. Furthermore, the weak limits of the truncated process can be identified along with some relevant properties. Finally, by using a measure-theoretic argument the weak convergence of the original process is established.

We remark that for the study of the one-particle problem one can assume less restrictive conditions. Namely, we can consider that the random field $F(\cdot)$ satisfies the following uniform mixing property. Let

$$
\tilde{\mathcal{G}}_{s}^{t}=\sigma\left\{F(u, \mathbf{y}, \cdot): s \leq u \leq t, \mathbf{y} \in \mathbb{R}^{m}\right\}
$$

and define the uniform mixing rate as

$$
\tilde{\phi}(s)=\sup _{r \geq 0} \sup \left\{|P(B)-P(B \mid A)|: A \in \tilde{\mathcal{G}}_{0}^{r}, B \in \tilde{\mathcal{G}}_{r+s}^{\infty}\right\} .
$$

We assume that

$$
\int_{0}^{\infty} s^{\gamma} \tilde{\phi}^{1 / 2}(s) d s<\infty, \quad \text { for } 0 \leq \gamma \leq 3
$$

In particular, if the condition C.3 presented early holds then the mixing condition (A.8) is satisfied.

Moreover, the condition C.5(a) is used in the proof only to ascertain the uniqueness of the corresponding diffusion process, and weaker conditions that assure this property can be found in [33].

A.2.1. Mixing Lemmas. For estimating some integrals in this appendix we will use two mixing lemmas which are slightly modified variants of Lemmas 1 and 2 in [27](pp. 109 and 112). 
Consider the random fields $U, V:[0,+\infty) \times \mathbb{R}^{n} \times \Omega \rightarrow \mathbb{R}$ that are strictly stationary and satisfy the continuity conditions

$$
\lim _{|\mathbf{h}| \rightarrow 0}\|U(s, \mathbf{h})-U(s, \mathbf{0})\|_{\infty}=\lim _{|\mathbf{h}| \rightarrow 0}\|V(s, \mathbf{h})-V(s, \mathbf{0})\|_{\infty}=0
$$

for any $s \geq 0$.

Lemma A.2. Assume that $U\left(\frac{\tau}{\epsilon}, \mathbf{y}\right)$ is $\tilde{\mathcal{G}}_{\tau / \epsilon}^{\infty}$-measurable and $E\left\{U\left(\frac{\tau}{\epsilon}, \mathbf{y}\right)\right\}=0$ for each fixed $\mathbf{y} \in \mathbb{R}^{n}$. Further, let $Z\left(\frac{\sigma}{\epsilon}\right), \sigma \leq \tau$ be a $\tilde{\mathcal{G}}_{0}^{\sigma / \epsilon}$-measurable random variable. Then for any $\varrho, 0 \leq \varrho \leq \sigma \leq \tau$, and a $\tilde{\mathcal{G}}_{0}^{\varrho / \epsilon}$-measurable random variable $\mathbf{y}_{\varrho}$ with values in $\mathbb{R}^{n}$,

$$
\left|E\left\{U\left(\frac{\tau}{\epsilon}, \mathbf{y}_{\varrho}\right) Z\left(\frac{\sigma}{\epsilon}\right)\right\}\right| \leq 2 \tilde{\phi}\left(\frac{\tau-\sigma}{\epsilon}\right)\left\|U\left(\frac{\tau}{\epsilon}, \cdot\right)\right\|_{\infty} E\left\{\left|Z\left(\frac{\sigma}{\epsilon}\right)\right|\right\} .
$$

The proof is standard and is based on a well-known mixing result of Ibragimov \& Linnik [26] and a simple approximation argument [3].

The following result is a useful variant of the previous lemma (see [26] for a proof).

Lemma A.3. Assume that $\left.U\left(\frac{\tau}{\epsilon}, \mathbf{y}\right), V\left(\frac{\sigma}{\epsilon}, \mathbf{y}\right)\right)$ are $\tilde{\mathcal{G}}_{\tau / \epsilon}^{\tau / \epsilon}$ and $\tilde{\mathcal{G}}_{\sigma / \epsilon}^{\sigma / \epsilon}$ measurable, respectively, for each fixed $\mathbf{y} \in \mathbb{R}^{n}$. Assume also that $E\left\{U\left(\frac{\tau}{\epsilon}, \mathbf{y}\right)\right\}=0$ and set

$$
W(\tau / \epsilon, \sigma / \epsilon, \mathbf{y})=E\{U(\tau / \epsilon, \mathbf{y}) V(\sigma / \epsilon, \mathbf{y})\} .
$$

Furthermore, let $Z\left(\frac{\varrho}{\epsilon}\right)$, $\mathbf{y}_{\varrho}$ be real and $\mathbb{R}^{n}$-valued, respectively, $\tilde{\mathcal{G}}_{0}^{\varrho / \epsilon}{ }^{-}$measurable random variables. Then for any $\varrho, 0 \leq \varrho \leq \sigma \leq \tau$,

$$
\begin{aligned}
\mid E\left\{Z\left(\frac{\varrho}{\epsilon}\right)\right. & {\left.\left[U\left(\frac{\tau}{\epsilon}, \mathbf{y}_{\varrho}\right) V\left(\frac{\sigma}{\epsilon}, \mathbf{y}_{\varrho}\right)-W\left(\frac{\tau}{\epsilon}, \frac{\sigma}{\epsilon}, \mathbf{y}_{\varrho}\right)\right]\right\} \mid \leq } \\
& 4 \tilde{\phi}^{\frac{1}{2}}\left(\frac{\tau-\sigma}{\epsilon}\right) \tilde{\phi}^{\frac{1}{2}}\left(\frac{\sigma-\varrho}{\epsilon}\right)\left\|U\left(\frac{\tau}{\epsilon}, \cdot\right)\right\|_{\infty}\left\|V\left(\frac{\sigma}{\epsilon}, \cdot\right)\right\|_{\infty} E\left\{\left|Z\left(\frac{\varrho}{\epsilon}\right)\right|\right\} .
\end{aligned}
$$

A.2.2. Truncated process. Let $M>\left|\mathbf{q}_{0}\right|$; consider a cutoff function $\varphi_{M}$ : $\mathbb{R}^{l} \rightarrow[0,1], \varphi_{M} \in C^{\infty}\left(\mathbb{R}^{l}\right)$ such that

$$
\varphi_{M}(\mathbf{q})=\left\{\begin{array}{ll}
0, & |\mathbf{q}| \geq 2 M \\
1, & |\mathbf{q}| \leq M
\end{array} ;\right.
$$

set $G^{M}(\mathbf{y}, \mathbf{q})=\varphi_{M}(\mathbf{q}) F(\mathbf{y})$, and define the truncated system of ODEs:

$$
\left\{\begin{array}{l}
\frac{d \mathbf{x}^{\epsilon, M}}{d s}=\tilde{\mathbf{q}}^{\epsilon, M} \\
\frac{d \mathbf{q}^{\epsilon, M}}{d s}=\frac{1}{\sqrt{\epsilon}} G^{M}\left(\frac{s}{\epsilon}, \frac{\mathbf{x}^{\epsilon, M}}{\epsilon^{a}}, \mathbf{q}^{\epsilon, M}\right)
\end{array}\right.
$$

with initial conditions $\left(\mathbf{x}_{0}, \mathbf{q}_{0}\right)$.

For each fixed $M$, one can prove the tightness of the family of measures $R^{\epsilon, M}$ induced by the truncated process $\mathbf{q}^{\epsilon, M}(s)$ in $\mathcal{D}^{l}=D\left([0, \infty) ; \mathbb{R}^{l}\right)$, the space of 'càdlàg' functions from $[0, \infty)$ to $\mathbb{R}^{l}$ with the Skorohod topology. 
The proof relies on the approach of [27](pp.107-108). It is enough to obtain that for $0 \leq t \leq u \leq T<\infty$ there exists $C=C(T)$ independent of $\epsilon \in(0,1]$ such that

$$
E\left\{\left|\mathbf{q}^{\epsilon, M}(u)-\mathbf{q}^{\epsilon, M}(t)\right|^{2} \Phi\right\} \leq C(u-t) E\{\Phi\}
$$

for $\Phi=\Phi(s, t)=\left|\mathbf{q}^{\epsilon, M}(t)-\mathbf{q}^{\epsilon, M}(s)\right|^{r}, 0 \leq s \leq t$ and $r=0,2$. This can be done by using an appropriate decomposition of $\left|\mathbf{q}^{\epsilon, M}(u)-\mathbf{q}^{\epsilon, M}(t)\right|^{2}$ and applying the mixing lemma A.2, as in [27] (the details are presented in [2]).

A.2.3. Limit identification. Our next step consists in the identification of the limit points of $R^{\epsilon}$. We proceed as in [27] by first identifying the limit points of $R^{\epsilon, M}$ in $\mathcal{D}^{l}$. Representing by $X(t)$ the $t$-coordinate function in $\mathcal{D}^{l}$, the corresponding $\sigma$-fields of subsets are given by $\mathcal{M}_{u}^{v}=\sigma\{X(t): u \leq t \leq v\}$.

Assume that $\left\{\epsilon_{n}\right\}$ is a sequence of positive numbers approaching zero such that the sequence of measures $\left\{R^{\epsilon_{n}, M}\right\}$ weakly converges to $R^{M}$ on $\mathcal{D}^{l}$ as $n \rightarrow \infty$. For any $f \in C^{\infty}\left(\mathbb{R}^{l}\right)$ with compact support define

$$
\mathcal{L}^{M} f(\mathbf{q})=\frac{1}{2} \sum_{i, j=1}^{l} A_{i j}^{M}(\mathbf{q}) \frac{\partial^{2} f}{\partial q_{i} \partial q_{j}}+\sum_{i=1}^{l} b_{i}^{M}(\mathbf{q}) \frac{\partial f}{\partial q_{j}},
$$

where

$$
\begin{aligned}
& A_{i j}^{M}(\mathbf{q})=\varphi_{M}^{2}(\mathbf{q}) \times \begin{cases}A_{i j}(\tilde{\mathbf{q}}), & \text { if } a=1 \\
A_{i j}(\mathbf{0}), & \text { if } 0<a<1,\end{cases} \\
& b_{i}^{M}(\mathbf{q})=\varphi_{M}(\mathbf{q}) \sum_{j=1}^{l} \begin{cases}\frac{\partial}{\partial q_{j}}\left(\varphi_{M}(\mathbf{q}) a_{i j}(\tilde{\mathbf{q}})\right), & \text { if } a=1 \\
\frac{\partial \varphi_{M}(\mathbf{q})}{\partial q_{j}} a_{i j}(\mathbf{0})+\varphi_{M}(\mathbf{q}) \frac{\partial a_{i j}}{\partial q_{j}}(\mathbf{0}), & \text { if } 0<a<1 .\end{cases}
\end{aligned}
$$

We can show that $f(X(t))-\int_{0}^{t} \mathcal{L}^{M} f(X(s)) d s$ is an $\left(R^{M}, \mathcal{M}_{0}^{t}\right)$ martingale. To establish this fact it suffices to prove that for any integer $n>0$, bounded continuous function $\Phi:\left(\mathbb{R}^{l}\right)^{n} \rightarrow \mathbb{R}$ and $0 \leq s_{1} \leq \cdots \leq s_{n} \leq t<u$, one has that

$$
\begin{aligned}
& E^{M}\left\{(f(X(u))-f(X(t))) \Phi\left(X\left(s_{1}\right), \ldots, X\left(s_{n}\right)\right)\right\}= \\
& E^{M}\left\{\int_{t}^{u} d s \mathcal{L}^{M} f(X(s)) \Phi\left(X\left(s_{1}\right), \ldots, X\left(s_{n}\right)\right)\right\},
\end{aligned}
$$

where $E^{M}\{\cdot\}$ represents expectation with respect to $R^{M}$. Observe, however, that because of the construction of $\mathcal{L}^{M}$ the measure $R^{M}$ is not uniquely determined by the above property.

Furthermore, since

$$
\begin{aligned}
I & =\lim _{n \rightarrow \infty} E\left\{\left(f\left(\mathbf{q}^{\epsilon_{n}, M}(u)\right)-f\left(\mathbf{q}^{\epsilon_{n}, M}(t)\right)\right) \Phi\left(\mathbf{q}^{\epsilon_{n}, M}\left(s_{1}\right), \ldots, \mathbf{q}^{\epsilon_{n}, M}\left(s_{n}\right)\right)\right\} \\
& =E^{M}\left\{(f(X(u))-f(X(t))) \Phi\left(X\left(s_{1}\right), \ldots, X\left(s_{n}\right)\right)\right\},
\end{aligned}
$$

it is enough to show that $I$ equals the left-hand side of (A.15).

This can be accomplished as in [27], see the details in [2]. We use an appropriate integral representation of $\left[f\left(\mathbf{q}^{\epsilon_{n}, M}(u)\right)-f\left(\mathbf{q}^{\epsilon_{n}, M}(t)\right)\right] \Phi$ as a sum containing the factors $\partial f\left(\mathbf{q}^{\epsilon_{n}, M}(\sigma)\right) / \partial q_{i}$ and $\partial^{2} f\left(\mathbf{q}^{\epsilon_{n}, M}(\sigma)\right) / \partial q_{i} \partial q_{j}$ in the integrand function. Then, by applying the mixing lemmas A.2 and A.3 and using the properties of the covariance functions $R_{i j}$, we are able to express the corresponding limiting expectation as an expectation with respect to the limiting measure $R^{M}$, resulting in an expression that coincides with the left-hand side of (A.15). 
A.2.4. Removal of cutoff and weak convergence. In this final step we remove the cutoff in $M$ and prove the weak convergence of the measures $R^{\epsilon}$. The argument is the same as in [27](step (vi), pp. 118-120), and we just highlight the main idea; see the details in [2].

From the continuity of $\mathbf{q}^{\epsilon_{n}, M}(s)$ and the properties of the coefficients $a_{i j}^{M}$ and $b_{i}^{M}$ it follows that the family of measures $\left\{R^{M}\right\}_{M \geq\left|\mathbf{q}_{0}\right|}$ is tight in $\mathcal{C}^{l}$.

Moreover, if a sequence $R^{M_{k}}$, with $M_{k} \rightarrow+\infty$ as $k \rightarrow+\infty$, converges weakly in $\mathcal{C}^{l}$ to some measure $R^{*}$, then it solves the martingale problem associated with $\mathcal{L}$. Thus, it is uniquely determined and we have that $R^{*}=R_{\mathbf{q}_{0}}$.

Finally, by using the same arguments as in [27](pp.119-120) we arrive to the conclusion that $R^{\epsilon}$ weakly converges to $R_{\mathbf{q}_{0}}$ in $\mathcal{C}^{l}$.

A.3. Two-particle problem. For any $0<\epsilon \leq 1$ define the stochastic process $\left(\mathbf{x}_{1}^{\epsilon}(s), \mathbf{q}_{1}^{\epsilon}(s), \mathbf{x}_{2}^{\epsilon}(s), \mathbf{q}_{2}^{\epsilon}(s)\right)$ with values in $\mathbb{R}^{m} \times \mathbb{R}^{l} \times \mathbb{R}^{m} \times \mathbb{R}^{l}$, for $s \geq 0$, as the solution of the system of ODEs

$$
\left\{\begin{array}{l}
\frac{d \mathbf{x}_{1}^{\epsilon}}{d s}=\tilde{\mathbf{q}}_{1}^{\epsilon} \\
\frac{d \mathbf{q}_{1}^{\epsilon}}{d s}=\frac{1}{\sqrt{\epsilon}} F\left(\frac{s}{\epsilon}, \frac{\mathbf{x}_{1}^{\epsilon}}{\epsilon^{a}}\right) \\
\frac{d \mathbf{x}_{2}^{\epsilon}}{d s}=\tilde{\mathbf{q}}_{2}^{\epsilon} \\
\frac{d \mathbf{q}_{2}^{\epsilon}}{d s}=\frac{1}{\sqrt{\epsilon}} F\left(\frac{s}{\epsilon}, \frac{\mathbf{x}_{2}^{\epsilon}}{\epsilon^{a}}\right) \\
\left(\mathbf{x}_{1}^{\epsilon}(0), \mathbf{q}_{1}^{\epsilon}(0), \mathbf{x}_{2}^{\epsilon}(0), \mathbf{q}_{2}^{\epsilon}(0)\right)=\left(\mathbf{x}_{10}, \mathbf{q}_{10}, \mathbf{x}_{20}, \mathbf{q}_{20}\right)
\end{array}\right.
$$

where $\left(\mathbf{x}_{10}, \mathbf{q}_{10}, \mathbf{x}_{20}, \mathbf{q}_{20}\right)$ is non-random.

Theorem A.4. Suppose that conditions C.1-C.5 (page 349) are fulfilled. Assume additionally that $m \geq 2$ and $\tilde{\mathbf{q}}_{10} \neq \tilde{\mathbf{q}}_{20}$. Then the stochastic process $\left(\mathbf{x}_{1}^{\epsilon}(s), \mathbf{q}_{1}^{\epsilon}(s), \mathbf{x}_{2}^{\epsilon}(s), \mathbf{q}_{2}^{\epsilon}(s)\right)$ converges weakly in $\mathcal{C}^{m+l} \times \mathcal{C}^{m+l}$ as $\epsilon \rightarrow 0$ to the process $\left(\mathbf{x}_{1}(s), \mathbf{q}_{1}(s), \mathbf{x}_{2}(s), \mathbf{q}_{2}(s)\right)$ such that

$$
\mathbf{x}_{\alpha}(s)=\mathbf{x}_{\alpha 0}+\int_{0}^{s} \tilde{\mathbf{q}}_{\alpha}\left(s^{\prime}\right) d s^{\prime}, \quad \alpha=1,2
$$

and $\mathbf{q}_{\alpha}(s), \alpha=1,2$ are two independent l-dimensional diffusions starting from $\mathbf{q}_{\alpha 0}$, $\alpha=1,2$, respectively. Furthermore, the generators $\mathcal{L}_{\mathbf{q}_{\alpha}}^{a}, \alpha=1,2$ corresponding to these diffusion processes are defined by (A.6).

The proof of the above theorem follows the same lines as in the previous section, the main difference being the fact that we need to keep the trajectories separated, in order to eliminate the mixed derivatives in the generator of the limiting diffusion process. This difficulty is overcome in the same way as in Theorem 4.4 from [3](p. 100).

We shall establish that the probability measure $R_{2}^{\epsilon}$ induced by the process $\left(\mathbf{q}_{1}^{\epsilon}(\cdot), \mathbf{q}_{2}^{\epsilon}(\cdot)\right)$ on $\mathcal{C}^{2 l}$ weakly converges to the measure $R_{2}=R_{\mathbf{q}_{10}} \otimes R_{\mathbf{q}_{20}}$, where $R_{\mathbf{q}_{\alpha 0}}$ is the probability measure on $\mathcal{C}^{l}$ associated with the diffusion starting from $\mathbf{q}_{\alpha 0}$ with generator $\mathcal{L}_{\mathbf{q}_{\alpha}}^{a}$.

We remark that some conditions of the theorem can be slightly relaxed. It suffices to consider that instead of condition C.3 we have the mixing condition (A.8) and in 
place of (A.4) (from condition C.4) to use the assumption

$$
\left|R_{i j}(\mathbf{y})\right| \leq C_{2} e^{-\frac{|\mathbf{y}|_{2}}{C_{2}}}, \quad \forall \mathbf{y} \in \mathbb{R}^{m+1}, \quad i, j=1, \ldots, l .
$$

We further consider the case where $\mathbf{x}_{10}=\mathbf{x}_{20}=\mathbf{0}$, remarking that in case this condition is not satisfied we can proceed in a similar way.

A.3.1. Truncated process and tightness. For the two-particles problem the truncated process is constructed in order to keep the trajectories bounded and separated.

Let $M>\left|\mathbf{q}_{\alpha 0}\right|, \quad \alpha=1,2$ and consider a cutoff function $\varphi_{M}: \mathbb{R}^{l} \rightarrow[0,1], \varphi_{M} \in$ $C^{\infty}\left(\mathbb{R}^{l}\right)$ such that

$$
\varphi_{M}(\mathbf{q})= \begin{cases}0, & |\mathbf{q}| \geq 2 M \\ 1, & |\mathbf{q}| \leq M\end{cases}
$$

Let $N>0$ and define $\tilde{Q}_{N}:=\left\{\left(\tilde{\mathbf{q}}_{1}^{\prime}, \tilde{\mathbf{q}}_{2}^{\prime}\right) \in \mathbb{R}^{2 m}:\left|\tilde{\mathbf{q}}_{j}^{\prime}-\tilde{\mathbf{q}}_{j 0}\right| \leq \frac{2}{N+1}, j=1,2\right\}$. Choose $N$ such that $\gamma_{N}:=\inf \left\{\left|\tilde{\mathbf{q}}_{1}^{\prime}-\tilde{\mathbf{q}}_{2}^{\prime}\right|:\left(\tilde{\mathbf{q}}_{1}^{\prime}, \tilde{\mathbf{q}}_{2}^{\prime}\right) \in \tilde{Q}_{N}\right\}>0$. We then have that the cones in $\mathbb{R}^{m+1}$ with vertex in the origin and basis $B_{\alpha}=\left\{(\mathbf{q}, 1):\left|\mathbf{q}-\mathbf{q}_{\alpha 0}\right| \leq \frac{1}{N+1}\right\}, \alpha=1,2$ are separated and consequently $\lambda_{N}>0$, where

$$
\lambda_{N}:=\inf \left\{\left|\mathbf{q}_{1}^{\prime}-\varrho \mathbf{q}_{2}^{\prime}\right| \wedge\left|\mathbf{q}_{2}^{\prime}-\varrho \mathbf{q}_{1}^{\prime}\right|: \mathbf{q}_{\alpha}^{\prime}=\left(\tilde{\mathbf{q}}_{\alpha}^{\prime}, 1\right), \alpha=1,2, \quad\left(\tilde{\mathbf{q}}_{1}^{\prime}, \tilde{\mathbf{q}}_{2}^{\prime}\right) \in \tilde{Q}_{N}\right\} .
$$

Let $p_{1}:=2^{q+2} p M, t^{(p)}=1 / p$ and $t_{k}^{\left(p_{1}\right)}=k / p_{1}, k=1,2, \ldots$. We introduce the smooth function $\psi_{N}: \mathbb{R}^{m} \rightarrow[0,1]$, with uniformly-in- $N$ bounded gradient and such that

$$
\psi_{N}(\tilde{\mathbf{q}})= \begin{cases}0, & \text { if }|\tilde{\mathbf{q}}| \geq \frac{2}{N+1} \\ 1, & \text { if }|\tilde{\mathbf{q}}| \leq \frac{1}{N+1}\end{cases}
$$

and the cutoff functions $\Psi_{\alpha}:[0, \infty) \times \mathbb{R}^{m} \rightarrow[0,1], \alpha=1,2$ :

$$
\Psi_{\alpha}(t, \tilde{\mathbf{q}})= \begin{cases}1, & \text { if } t \geq t^{(p)} \\ \psi_{N}\left(\tilde{\mathbf{q}}-\tilde{\mathbf{q}}_{\alpha 0}\right), & \text { if } 0 \leq t<t^{(p)} .\end{cases}
$$

Further, we consider the functions $\xi_{k}: \mathbb{R}^{m} \times \mathcal{D}^{m} \rightarrow[0,1]$ smooth when the path $\tilde{X}(\cdot) \in$ $\mathcal{D}^{m}$ is fixed and such that

$$
\xi_{k}(\mathbf{y} ; \tilde{X}(\cdot))= \begin{cases}1, & \text { if }\left|\mathbf{y}-\int_{0}^{t_{k}^{\left(p_{1}\right)}} \tilde{X}(s) d s\right| \geq \frac{2}{q} \\ 0, & \text { if }\left|\mathbf{y}-\int_{0}^{t_{k}^{\left(p_{1}\right)}} \tilde{X}(s) d s\right| \leq \frac{1}{q}\end{cases}
$$

and introduce the cutoff function $\Xi:[0, \infty) \times \mathbb{R}^{m} \times \mathcal{D}^{m} \rightarrow[0,1]$ :

$$
\Xi(t, \mathbf{y} ; \tilde{X}(\cdot))= \begin{cases}1, & \text { if } 0 \leq t<t^{(p)} \\ \xi_{k}(\mathbf{y} ; \tilde{X}(\cdot)) & \text { if } t_{k}^{\left(p_{1}\right)} \leq t<t_{k+1}^{\left(p_{1}\right)} \text { where } t_{k}^{\left(p_{1}\right)} \geq t^{(p)} .\end{cases}
$$

We finally sum up the effect of all these cutoff functions by defining $\Theta_{\alpha}:[0, \infty) \times \mathbb{R}^{m} \times$ $\mathbb{R}^{l} \times \mathcal{D}^{m} \rightarrow[0,1], \alpha=1,2:$

$$
\Theta_{\alpha}(t, \mathbf{y}, \mathbf{q} ; \tilde{X}(\cdot))=\varphi_{M}(\mathbf{q}) \Psi_{\alpha}(t, \tilde{\mathbf{q}}) \Xi(t, \mathbf{y} ; \tilde{X}(\cdot)) .
$$


Let

$$
G_{\alpha}^{\epsilon, M, N, p, q}(t, \mathbf{y}, \mathbf{q} ; \tilde{X}(\cdot))=\Theta_{\alpha}\left(\epsilon t, \epsilon^{a} \mathbf{y}, \mathbf{q} ; \tilde{X}(\cdot)\right) F(t, \mathbf{y})
$$

and define the truncated system of ODEs:

$$
\left\{\begin{array}{l}
\frac{d \overline{\mathbf{x}}_{1}^{\epsilon}}{d s}=\tilde{\mathbf{q}}_{1}^{\epsilon} \\
\frac{d \overline{\mathbf{q}}_{1}^{\epsilon}}{d s}=\frac{1}{\sqrt{\epsilon}} G_{1}^{\epsilon, \tilde{M}}\left(\frac{s}{\epsilon}, \frac{\overline{\mathbf{x}}_{1}^{\epsilon}}{\epsilon^{a}}, \overline{\mathbf{q}}_{1}^{\epsilon}\right) \\
\frac{d \overline{\mathbf{x}}_{2}^{\epsilon}}{d s}=\tilde{\mathbf{q}}_{2}^{\epsilon} \\
\frac{d \overline{\mathbf{q}}_{2}^{\epsilon}}{d s}=\frac{1}{\sqrt{\epsilon}} G_{2}^{\epsilon, \tilde{M}}\left(\frac{s}{\epsilon}, \frac{\overline{\mathbf{x}}_{2}^{\epsilon}}{\epsilon^{a}}, \overline{\mathbf{q}}_{2}^{\epsilon}\right)
\end{array}\right.
$$

with initial conditions $\left(\mathbf{x}_{10}, \mathbf{q}_{10}, \mathbf{x}_{20}, \mathbf{q}_{20}\right)$, where

$$
\begin{aligned}
& G_{1}^{\epsilon, \tilde{M}}(t, \mathbf{y}, \mathbf{q})=G_{1}^{\epsilon, M, N, p, q}\left(t, \mathbf{y}, \mathbf{q} ; \tilde{\mathbf{q}}_{2}^{\epsilon}(\cdot)\right), \\
& G_{2}^{\epsilon, \tilde{M}}(t, \mathbf{y}, \mathbf{q})=G_{2}^{\epsilon, M, N, p, q}\left(t, \mathbf{y}, \mathbf{q} ; \tilde{\tilde{\mathbf{q}}}_{1}^{\epsilon}(\cdot)\right) .
\end{aligned}
$$

Note the simplifying notation $\tilde{M}=(M, N, p, q)$.

We remark that the main differences between the cutoff function defined in Section A.2 and the function $\Theta_{\alpha}$ defined above are that the latter depends on time $t$, both variables $\mathbf{y}, \mathbf{q}$ and also, parametrically, on a path from $\mathcal{D}^{m}$, while the former only depends on $\mathbf{q}$. Furthermore, we notice that when $0 \leq t<t^{(p)}$ or $t_{k}^{\left(p_{1}\right)} \leq t<t_{k+1}^{\left(p_{1}\right)}$ and the path remain fixed, $\Theta_{\alpha}$ is independent of $t$ and smooth on the other arguments.

For each fixed $\tilde{M}=(M, N, p, q)$, the tightness of the family of measures $R_{2}^{\epsilon, \tilde{M}}$, $0<\epsilon \leq 1$ induced by the truncated process $\left(\overline{\mathbf{q}}_{1}^{\epsilon}(s), \overline{\mathbf{q}}_{2}^{\epsilon}(s)\right)$ in $\mathcal{D}^{2 l}=D\left([0, \infty) ; \mathbb{R}^{2 l}\right)$ follows (after minor changes) as in Section A.2, since we only need to establish the bound (A.13) for $0 \leq t<u \leq t^{(p)}$ or $t_{k}^{\left(p_{1}\right)} \leq t<u \leq t_{k+1}^{\left(p_{1}\right)}$ with $t^{(p)} \leq t_{k}^{\left(p_{1}\right)}$.

A.3.2. Limit identification. As in the case of one particle, our next step consists in the identification of the limit points of the family of measures $R_{2}^{\epsilon, \tilde{M}}$, as $\epsilon \rightarrow 0$. We represent by $X(t)=\left(X_{1}(t), X_{2}(t)\right)$ the $t$-coordinate function in $\mathcal{D}^{2 l}$ and let

$$
Y_{\alpha}(t)=\int_{0}^{t} \tilde{X}_{\alpha}(s) d s, \quad \alpha=1,2 .
$$

For any $f \in C_{0}^{\infty}\left(\mathbb{R}^{2 l}\right)$ define the operator

$$
\begin{aligned}
\left(\mathcal{L}^{\tilde{M}} f\right)\left(\mathbf{q}_{1}, \mathbf{q}_{2} ; \tilde{X}(\cdot)\right)=\sum_{\alpha=1,2}\left\{\frac{1}{2} \sum_{i, j=1}^{l} A_{\alpha i j}^{\tilde{M}}\left(s, \mathbf{q}_{\alpha} ; \tilde{X}(\cdot)\right) \frac{\partial^{2} f}{\partial q_{i}^{\alpha} \partial q_{j}^{\alpha}}\right. \\
\left.+\sum_{i=1}^{l} b_{\alpha i}^{\tilde{M}}\left(s, \mathbf{q}_{\alpha} ; \tilde{X}(\cdot)\right) \frac{\partial f}{\partial q_{j}^{\alpha}}\right\}
\end{aligned}
$$

with the coefficients defined as follows:

$$
A_{\alpha i j}^{\tilde{M}}(s, \mathbf{q} ; \tilde{X}(\cdot))=\Lambda_{\alpha}^{2}(s, \mathbf{q} ; \tilde{X}(\cdot)) \times \begin{cases}A_{i j}(\tilde{\mathbf{q}}), & \text { if } a=1 \\ A_{i j}(\mathbf{0}), & \text { if } 0<a<1,\end{cases}
$$




$$
\begin{array}{rlrl}
b_{\alpha i}^{\tilde{M}}(s, \mathbf{q} ; \tilde{X}(\cdot)) & =\Lambda_{\alpha}(s, \mathbf{q} ; \tilde{X}(\cdot)) & \\
& \times \sum_{j=1}^{l} \begin{cases}\frac{\partial}{\partial q_{j}}\left(\Lambda_{\alpha}(s, \mathbf{q} ; \tilde{X}(\cdot)) a_{i j}(\tilde{\mathbf{q}})\right) & \text { if } a=1 \\
\frac{\partial}{\partial q_{j}} \Lambda_{\alpha}(s, \mathbf{q} ; \tilde{X}(\cdot)) a_{i j}(\mathbf{0})+\Lambda_{\alpha}(s, \mathbf{q} ; \tilde{X}(\cdot)) \frac{\partial a_{i j}}{\partial q_{j}}(\mathbf{0}) & \text { if } 0<a<1,\end{cases}
\end{array}
$$

where

$$
\Lambda_{\alpha}(s, \mathbf{q} ; \tilde{X}(\cdot))=\Theta_{\alpha}\left(s, \mathbf{q}, Y_{\alpha}(s) ; \tilde{X}_{\hat{\alpha}}(\cdot)\right) \quad \text { and } \quad \hat{\alpha}= \begin{cases}1, & \text { if } \alpha=2 \\ 2, & \text { if } \alpha=1\end{cases}
$$

We can establish a martingale property for any limiting measure $R_{2}^{\tilde{M}}$. It is enough to prove that for any integer $n>0$, bounded continuous function $\Phi:\left(\mathbb{R}^{2 l}\right)^{n} \rightarrow \mathbb{R}$ and $0 \leq s_{1} \leq \cdots \leq s_{n} \leq t<u$

$$
\begin{aligned}
E_{2}^{\tilde{M}}\left\{(f(X(u))-f(X(t))) \Phi\left(X\left(s_{1}\right), \cdots, X\left(s_{n}\right)\right)\right\} \\
=E_{2}^{\tilde{M}}\left\{\int_{t}^{u}\left(\mathcal{L}^{\tilde{M}} f\right)(X(s) ; \tilde{X}(\cdot)) \Phi\left(X\left(s_{1}\right), \cdots, X\left(s_{n}\right)\right),\right\}
\end{aligned}
$$

where $E_{2}^{\tilde{M}}\{\cdot\}$ represents expectation with respect to $R_{2}^{\tilde{M}}$. As has been remarked in [28], it is sufficient to consider the case where $0 \leq t<u \leq t^{(p)}$ or $t_{k}^{\left(p_{1}\right)} \leq t<u \leq t_{k+1}^{\left(p_{1}\right)}$ with $t_{k}^{\left(p_{1}\right)} \geq t^{(p)}$.

The calculations for obtaining the martingale property are very similar to those described in Section A.2. We have to determine the limit

$$
\begin{aligned}
I & =E_{2}^{\tilde{M}}\left\{(f(X(u))-f(X(t))) \Phi\left(X\left(s_{1}\right), \ldots, X\left(s_{n}\right)\right)\right\} \\
& =\lim _{n \rightarrow \infty} E\left\{\left(f\left(\overline{\mathbf{q}}^{\epsilon_{n}}(u)\right)-f\left(\overline{\mathbf{q}}^{\epsilon_{n}}(t)\right)\right) \Phi\left(\overline{\mathbf{q}}^{\epsilon_{n}}\left(s_{1}\right), \ldots, \overline{\mathbf{q}}^{\epsilon_{n}}\left(s_{n}\right)\right)\right\},
\end{aligned}
$$

where the sequence $\left\{\epsilon_{n}\right\}$ is such that $R_{2}^{\epsilon_{n}, \tilde{M}}$ weakly converges to $R_{2}^{\tilde{M}}$ when $\epsilon_{n} \rightarrow 0$, as $n \rightarrow+\infty$.

This can be accomplished as in Section A.2 by using an appropriate integral representation for the difference $\left(f\left(\overline{\mathbf{q}}^{\epsilon_{n}}(u)\right)-f\left(\overline{\mathbf{q}}^{\epsilon_{n}}(t)\right)\right) \Phi$ and the mixing lemmas A.2 and A.3. But now there will be additional factors of the form $\partial^{2} f\left(\overline{\mathbf{q}}^{\epsilon_{n}}(\sigma)\right) / \partial q_{i}^{\alpha} \partial q_{j}^{\beta}$ with $\alpha \neq \beta$ in the integrand function. In the limit we can get rid of those terms, since the covariance functions $R_{i j}$ decay exponentially fast and the two trajectories remain sufficiently separated as we enforce through the introduced truncation.

Finally, we obtain that $I$ can be expressed as an expectation with respect to the limiting measure $R_{2}^{\tilde{M}}$. The resulting expression coincides with the left-hand side of A.19, hence the martingale property follows.

A.3.3. Removal of cutoff and weak convergence. In this final step, we shall remove the cutoff and establish the weak convergence of $\left(\mathbf{q}_{1}^{\epsilon}(\cdot), \mathbf{q}_{2}^{\epsilon}(\cdot)\right)$. We will follow the same strategy as presented in $[3,28]$, so we just highlight the main ideas; the details can be found in [2].

We consider that all measures are supported in the corresponding space of continuous functions and that the convergence also takes place in that space. This property can be established as in [27].

Let us define a stopping time $U(\cdot ; M, N, p, q)$ with the property that the dynamics of the truncated and original systems coincide up to this time. Moreover, this 
property allows us to identify any limit measure $R_{2}^{\tilde{M}}$ with $R_{2}$ on the $\sigma$-algebra corresponding to the stopping time. Finally, by choosing sufficiently large $M, N$ we get that $U(\cdot ; M, N, p, q)$ converges to infinity as $q \rightarrow \infty$ and $p \rightarrow \infty$; from this the weak convergence follows by the same calculations as in [3].

Let $X(\cdot)=\left(X_{1}(\cdot), X_{2}(\cdot)\right) \in \mathcal{C}^{2 l}$ and $Y_{\alpha}(t)=\int_{0}^{t} \tilde{X}_{\alpha}(s) d s$. For such $X(\cdot)$ we define the following stopping times:

$$
\begin{aligned}
S(N, p) & :=\lim _{n \rightarrow \infty} S_{n}(N, p) \\
T(M) & :=\lim _{n \rightarrow \infty} T_{n}(M) \\
V(p, q) & :=\lim _{n \rightarrow \infty} V_{n}(p, q),
\end{aligned}
$$

where

$$
\begin{aligned}
S_{n}(N, p) & =\inf \left\{t: 0 \leq t<t^{(p)},\left|\tilde{X}_{\alpha}(t)-\tilde{\mathbf{q}}_{\alpha 0}\right|>\frac{1}{N+1}-\frac{1}{n} \quad \text { for } \alpha=1 \text { or } 2\right\}, \\
T_{n}(M) & =\inf \left\{t \geq 0:\left|X_{\alpha}(t)\right|>M-\frac{1}{n} \quad \text { for } \alpha=1 \text { or } 2\right\}, \\
V_{n}(p, q) & =\inf \left\{t \geq t^{(p)},\left|Y_{1}(t)-Y_{2}(t)\right|<\frac{9}{4 q}+\frac{1}{n}\right\} .
\end{aligned}
$$

We also adopt the convention that if the corresponding set of times is empty then the stopping time is infinite.

Let $T_{0}>0$ and set

$$
U(N, M, p, q):=\{S(N, p) \wedge T(M) \wedge V(p, q)\}
$$

and

$$
B(N, M, p, q):=\left\{S(N, p) \wedge V(p, q) \leq T(M) \wedge T_{0}\right\} ;
$$

note that $B \in \mathcal{M}_{0}^{U}$ (the $\sigma$-algebra generated by the stopping time $U$ ). Moreover, we have that

$$
T_{0}<U\left(\left(\overline{\mathbf{q}}_{1}^{\epsilon}(\cdot), \overline{\mathbf{q}}_{2}^{\epsilon}(\cdot)\right) ; N, M, p, q\right)
$$

implies that $\left(\overline{\mathbf{q}}_{1}^{\epsilon}(s), \overline{\mathbf{q}}_{2}^{\epsilon}(s)\right)=\left(\mathbf{q}_{1}^{\epsilon}(s), \mathbf{q}_{2}^{\epsilon}(s)\right)$ for $s \in\left[0, T_{0}\right]$.

The following results will be useful for establishing the weak convergence. We begin with two simple lemmas:

Lemma A.5. We have that $\lim _{M \rightarrow \infty} T(M)=+\infty$, a.s. $R_{2}$.

Lemma A.6. We have that $\lim _{p \rightarrow \infty} S(N, p)=+\infty, \forall N$ a.s. $R_{2}$.

These lemmas are direct consequences of the continuity of the paths of the limiting diffusion process.

The next lemma shows that $S(N, p) \wedge V(p, q)$ becomes infinity as $q \rightarrow+\infty, p \rightarrow+\infty$ (in this order).

Lemma A.7. For $N$ sufficiently large and $T_{1}, \eta>0$ arbitrary, one can find $p_{0}$ such that $R_{2}\left\{S(\cdot ; N, p) \wedge V(\cdot ; p, q) \leq T_{1}\right\} \leq \eta$ for any $p \geq p_{0}$ and $q \geq q_{0}(p)$.

The proof is based on Lemma A.6, the continuity of the paths and the hypoellipticity of the diffusion $\left(\int_{0}^{t}\left(\tilde{X}_{1}(s)-\tilde{X}_{2}(s)\right) d s, X(t)\right)$ (see details in [2]). 
A straightforward and useful consequence of the lemmas above is given by the next corollary.

Corollary A.8. For any $\eta>0$, there exist sufficiently large $M, N, p$ and $q$ such that

$$
R_{2}\{B(\cdot ; M, N, p, q)\} \leq \eta .
$$

Now with the aid of the lemmas above we can establish as in [3] that for any $T_{0}>0$ and a continuous and bounded functional $H$ on $\mathcal{C}^{2 l}$ that is $\mathcal{M}_{0}^{T_{0}}$-measurable, we have that

$$
\limsup _{\epsilon \rightarrow 0} E\left\{H\left(\mathbf{q}_{1}^{\epsilon}(\cdot), \mathbf{q}_{2}^{\epsilon}(\cdot)\right)\right\} \leq \int H(X) R_{2}\{d X\} .
$$

The proof of this fact is just a repetition of the calculations in [3](pp.126-127). Finally, using the same argument presented in [27], the proof of the weak convergence of $R_{2}^{\epsilon}$ to $R_{2}=R_{\mathbf{q}_{10}} \otimes R_{\mathbf{q}_{20}}$ can be completed.

\section{REFERENCES}

[1] D.G. Alfaro Vigo, J.P. Fouque, J. Garnier and A. Nachbin, Robustness of time reversal for waves in time-dependent random media, Stochastic Process. Appl., 113(2), 289-313, 2004.

[2] D.G. Alfaro Vigo and K. Sølna, Time reversal of waves in a perturbed random medium, preprint, 2007. (http://www.preprint.impa.br/Shadows/SERIE $\sqcup$ A/2007/529.html)

[3] G. Bal, T. Komorowski and L. Ryzhik, Self-averaging of Wigner transforms in random media, Comm. Math. Phys., 242(1-2), 81-135, 2003.

[4] G. Bal, G. Papanicolaou and L. Ryzhik, Self-averaging in time reversal for the parabolic wave equation, Stoch. Dyn., 2, 507-531, 2002.

[5] G. Bal and L. Ryzhik, Time reversal for classical waves in random media, C.R. Acad. Sci. Paris, 333, 1041-1046, 2001.

[6] G. Bal and L. Ryzhik, Time reversal and refocusing in random media, SIAM J. Appl. Math., 63(5), 1475-1498, 2003.

[7] G. Bal and L. Ryzhik, Stability of time reversed waves in changing media, Discrete Contin. Dyn. Syst., 12(5), 793-815, 2005.

[8] G. Bal and L. Ryzhik, Wave field correlations in weakly mismatched random media, Stoch. Dyn., 6(3), 301-328, 2006.

[9] G. Bal and R. Verástegui, Time-reversal in changing environments, Multiscale Model. Simul., 2(4), 639-661, 2004.

[10] A. Bamberger, E. Engquist, L. Halpern and P. Joly, Parabolic wave equation approximations in heterogeneous media, SIAM J. Appl. Math., 48, 99-128, 1988.

[11] P. Blomgren, G. Papanicolaou and H. Zhao, Super-resolution in time-reversal acoustics, J. Acoust. Soc. Amer., 111, 230-248, 2002.

[12] A. Derode, A. Tourin, J. De Rosny, M. Tanter, S. You and M. Fink, Taking advantadge of multiple scattering to communicate with time reversal antennas, Phys. Rev. Lett., 90, 014-301, 2001.

[13] D. Dowling and D. Jackson, Phase conjugation in underwater acoustics, J. Acoust. Soc. Amer., 89, 171-181, 1990.

[14] D. Dowling and D. Jackson, Narrow-band performance of phase-conjugate arrays in dynamic random media, J. Acoust. Soc. Amer., 91, 3257-3277, 1992.

[15] G.F. Edelman, T. Akal, W.S. Hodgkiss, S. Kim, W.A. Kuperman and H.C. Song, An initial demonstration of underwater acoustic communications using time reversal, IEEE J. Oceanic Eng., 27, 602-609, 2002.

[16] A. Fannjiang, White-noise and geometrical optics limits of Wigner-Moyal equation for wave beams in turbulent media, Comm. Math. Phys., 254, 289-322, 2005.

[17] A. Fannjiang and K. Sølna, Propagation and time-reversal of wave beams in atmospheric turbulence, SIAM Multiscale Model. Simul., 3(3), 522-558, 2005.

[18] A. Fannjiang and K. Sølna, Superresolution and duality for time-reversal of waves in random media, Phys. Lett. A, 352(1-2), 22-29, 2005. 
[19] A. Fannjiang and K. Sølna, Time reversal of parabolic waves and two-frequency Wigner distribution, Discrete Cont. Dyn. Syst.-B, 6(4), 783-802, 2006.

[20] M. Fink and J. de Rosny, Time-reversed acoustics in random media and in chaotic cavities, Nonlinearity, 15, R1-R18, 2002.

[21] M. Fink and C. Prada, Acoustic time-reversal mirrors, Inverse Problems, 17(1), R1-R38, 2001.

[22] J.P. Fouque, J. Garnier, G. Papanicolaou and K. Solna, Wave Propagation and Time Reversal in Randomly Layered Media, Springer, 2007.

[23] J.P. Fouque, J. Garnier and K. Sølna, Time reversal super resolution in randomly layered media, Wave Motion, 42, 238-260, 2006.

[24] P. Gérard, P. Markovich, N. Mauser and F. Poupaud, Homogenization limits and Wigner transforms, Comm. Pure Appl. Math., 50, 323-380, 1997.

[25] W.S. Hodgkiss, H.C. Song, W.A. Kuperman, T. Akal, C. Ferla and D.R. Jackson, A long range and variable focus phase-conjugation experiment in a shallow water, J. Acoust. Soc. Amer., 105, 1597-1604, 1999.

[26] I.A. Ibragimov and Yu.V. Linnik, Independent and Stationary Sequences of Random Variables, Wolters-Noordhoff Publishing, Groningen, 1971.

[27] H. Kesten and G. Papanicolaou, A limit theorem for turbulent diffusion, Comm. Math. Phys., 65, 97-128, 1979.

[28] H. Kesten and G. Papanicolaou, A limit theorem for stochastic acceleration, Comm. Math. Phys., 78, 19-63, 1980.

[29] P.-L. Lions and T. Paul, Sur les measures de Wigner, Rev. Mat. Iberoamericana, 9(3), 553-618, 1993.

[30] G. Papanicolaou, L. Ryzhik and K. Sølna, The parabolic wave approximation and time reversal, Mat. Contemp., 23, 139-159, 2002.

[31] G. Papanicolaou, L. Ryzhik and K. Sølna, Statistical stability in time reversal, SIAM J. Appl. Math., 64, 1133-1155, 2004.

[32] G. Papanicolaou, L. Ryzhik and K. Sølna, Self-averaging from lateral diversity in the ItôSchrödinger equation, Multiscale Model. Simul., accepted, 2007.

[33] D. Stroock and S.R.S. Varadhan, Multidimensional Diffusion Processes, Springer-Verlag, Berlin, New York, 1979.

[34] A. Tourin, A. Derode and M. Fink, Sensitivity to perturbations of a time-reversed acoustic wave in a multiple scattering medium, Phys. Rev. Lett., 87(27), 4301, 2001. 